\title{
Adaptive terminal sliding mode control of a rigid robotic manipulator with uncertain dynamics incorporating constraint inequalities
}

\author{
P. G. Keleher* $\quad$ R. J. Stonier ${ }^{\dagger}$
}

Received 24 April, 2001; revised 21 January, 2002

\begin{abstract}
We consider the control of a rigid robotic manipulator using robust adaptive sliding mode tracking control. Physical state constraints are
\end{abstract}

* School of Industrial Ecology and the Built Environment, James Goldston Faculty of Engineering and Physical Systems, Central Queensland University, Rockhampton, Qld 4702, Australia. mailto:p.keleher@cqu.edu.au

${ }^{\dagger}$ School of Mathematics and Decision Science, Faculty of Informatics and Communications, Central Queensland University, Rockhampton, Qld 4702, Australia. mailto:r.stonier@cqu.edu.au

${ }^{0}$ See http: //anziamj . austms . org.au/V43/E039 for this article and ancillary services, (C) Austral. Mathematical Soc. 2002. Published March 25, 2002. 
incorporated using a multiplicative penalty in a Liapunov function from which we obtain analytic control laws that drive the robot's end effector into a desired fixed target within finite time.

\section{Contents}

1 Introduction

E104

1.1 Incorporating constraints . . . . . . . . . . . . E105

2 General analysis

E107

3 Control law calculations

E109

4 Applications to a 2 link manipulator

E115

5 Simulation discussion

E119

5.1 Simulation A: tracking a fixed point reference signal . . . E120

5.2 Simulation B: tracking a fixed point reference signal . . . . E129

5.3 Simulation C: tracking a fixed point reference signal . . . E136

5.4 Simulation D: tracking an oscillatory reference signal . . . E137

6 Conclusions

E151

References

E151 


\section{Introduction}

The application of Liapunov theory is a fundamental tool used in non-linear control theory $[4,5]$. Various forms of Liapunov stability are used to ensure that the system under control is (i) stable and (ii) convergence to desired behaviour is achieved by asymptotic stability (convergence in infinite time) and finite attraction (convergence in finite time).

Consider the autonomous system whose dynamics are written in normal form

$$
\frac{d \boldsymbol{x}}{d t}=\boldsymbol{f}(\boldsymbol{x}, \boldsymbol{u})
$$

with state $\boldsymbol{x} \in R^{n}$ and control $\boldsymbol{u} \in R^{m}$.

Suppose we determine a Liapunov function $V: R^{n} \rightarrow R^{n}$, which is positive definite at a particular state $\boldsymbol{x}_{e}$, that is $V\left(\boldsymbol{x}_{e}\right)=0$ and $V(\boldsymbol{x})>0$ for all $\boldsymbol{x} \neq \boldsymbol{x}_{e}$. The idea behind the Liapunov method is to find a control law $\boldsymbol{u}$ which makes the total derivative of $V$

$$
\dot{V}(t)=\nabla V^{T}(\boldsymbol{x}) \frac{d \boldsymbol{x}}{d t}=\nabla V^{T}(\boldsymbol{x}) \boldsymbol{f}(\boldsymbol{x}, \boldsymbol{u}),
$$

satisfy an appropriate negative condition, thus ensuring that $V$ decreases appropriately on trajectories of the system under control. These conditions on $V$ ensure that the state $\boldsymbol{x} \rightarrow \boldsymbol{x}_{e}$ as $t \rightarrow \infty$. It is usual to select $\boldsymbol{x}_{e}$ as an equilibrium state of Equation (1). 
As outlined by [3] and Chapter 3 of [5] the use of the concept of asymptotic stability for control of the system to a desired equilibrium, requires that $\dot{V}$ be made negative definite in some region about the equilibrium. This method ensures that the desired equilibrium is reached in "infinite" time.

To control the state of the system to a switching surface in state space, and then constrain the system dynamics so that it will remain on the switching surface until the desired target or equilibrium is reached is fundamental to what is called "sliding mode" control [5]. It uses the concept of finite attraction with so called "terminal attractors" [10]. This theory requires the control to be selected in such a way that the derivative of an appropriate Liapunov function $V$ is more strongly negative definite than in asymptotic stability so that finite attraction is achieved.

\subsection{Incorporating constraints}

Many results obtained in the control of physical systems do not account for physical constraints that are imposed on the state of the system. One way to add the constraints using Liapunov theory is to incorporate them as penalty terms in the Liapunov function $[2,6]$.

In [9], finite time switching control of two planar PR manipulators operating in the same workspace was investigated, which involved physical constraints and obstacles being incorporated in the Liapunov analysis as added penalty terms. For example, the Liapunov function for Arm 1 was defined 
as:

$$
V^{1}=V_{0}^{1}+\sum_{i=1}^{8} \frac{\beta_{1}[i]}{V_{i}^{1}},
$$

where $\beta_{1}[i],(i=1, \cdots, 8)$ were positive constants associated with the functions $V_{i}$ defined by 8 constraints. The function $V_{0}^{1}$ measured the displacement of Robot 1 from a target state $\mathcal{T}_{1}$ in Cartesian coordinates. This term assured that there was attraction to the target under continued decrease of the Liapunov function. Physical constraints and obstacles were incorporated additively in the second term of $V^{1}$ as "penalty" terms, using the language of penalties from constrained optimisation theory. The selected positive penalty parameters $\beta_{1}[i]$ acted as avoidance parameters and the $V_{i}^{1}$ functions defined the physical constraints or obstacles to be avoided. For example, the function

$$
V_{2}^{1}=1.5-x_{2}
$$

corresponded to the constraint on variable $x_{2}$, which was that $x_{2} \leq 1.5$. For constraint satisfaction it is required that $V_{2}>0$. Here the region

$$
\mathcal{A T}_{2}^{1}=\left\{\boldsymbol{x} \mid x_{2}-1.5 \geq 0\right\}
$$

was considered to be an avoidance region and $V_{2}^{1}>0$ on the complement of $\mathcal{A T}_{2}^{1}$.

In [7] a Liapunov function was developed that incorporated constraints as multiplicative penalties in the Liapunov function. It was used to formulate strategies to control simulated point mass mobile robots to desired targets 
in finite time. Sliding mode was used in preference to asymptotic stability concepts. A similar analysis was used in [8] to show that this method could be used to control a prismatic-revolute robot arm in the presence of state constraints to a desired target.

In this paper we extend the theory and application to a rigid robotic manipulator [12] in which the system dynamics has "uncertainty" in various system parameters and requires control to track a desired reference trajectory.

Our objective is to develop the adaptive sliding mode control of such a robotic system for model reference tracking, incorporating physical constraints through a multiplicative term in an appropriate Liapunov function.

Furthermore we desire the state to track a desired trajectory with time. This is typically referred to as Model Reference Adaptive Control under uncertainty.

\section{General analysis}

We begin with a general analysis of an n-joint rigid robotic manipulator system whose dynamics may be described by the second-order nonlinear vector differential equation, [12]:

$$
M(\boldsymbol{q}) \ddot{\boldsymbol{q}}+h(\boldsymbol{q}, \dot{\boldsymbol{q}})=\boldsymbol{u}(t)+\boldsymbol{d}(t),
$$


where $\dot{\boldsymbol{q}}(t)$ is the $n \times 1$ vector of joint angular positions, $M(\boldsymbol{q})$ is the $n \times n$ symmetric positive definite inertia matrix, $\boldsymbol{h}(\boldsymbol{q}, \dot{\boldsymbol{q}})$ is the $n \times 1$ vector containing Coriolis, centrifugal forces and gravity torques, $\boldsymbol{u}(t)$ is the $n \times 1$ vector of applied joint torques (control inputs) and $\boldsymbol{d}(t)$ is the $n \times 1$ vector of the bounded input disturbances. Assuming that the system described by (2) has parts which are known $M_{o}(\boldsymbol{q}), \boldsymbol{h}_{o}(\boldsymbol{q}, \dot{\boldsymbol{q}})$ and unknown $\Delta M(\boldsymbol{q}), \Delta \boldsymbol{h}(\boldsymbol{q}, \dot{\boldsymbol{q}})$, then

$$
\begin{aligned}
M(\boldsymbol{q}) & =M_{o}(\boldsymbol{q})+\Delta M(\boldsymbol{q}), \\
\boldsymbol{h}(\boldsymbol{q}, \dot{\boldsymbol{q}}) & =\boldsymbol{h}_{o}(\boldsymbol{q}, \dot{\boldsymbol{q}})+\Delta \boldsymbol{h}(\boldsymbol{q}, \dot{\boldsymbol{q}}) .
\end{aligned}
$$

The dynamic equation may be rewritten as

$$
M_{o}(\boldsymbol{q}) \ddot{\boldsymbol{q}}+\boldsymbol{h}_{o}(\boldsymbol{q}, \dot{\boldsymbol{q}})=\boldsymbol{u}(t)+\boldsymbol{\rho}(t),
$$

where the system uncertainty, $\boldsymbol{\rho}(t)$, is defined to be

$$
\boldsymbol{\rho}(t)=-\Delta M(\boldsymbol{q}) \ddot{\boldsymbol{q}}-\Delta \boldsymbol{h}(\boldsymbol{q}, \dot{\boldsymbol{q}})+\boldsymbol{d}(t) .
$$

A "nominal system" which has no uncertainty is defined as

$$
M_{o}(\boldsymbol{q}) \ddot{\boldsymbol{q}}+\boldsymbol{h}_{o}(\boldsymbol{q}, \dot{\boldsymbol{q}})=\boldsymbol{u}_{1}(t) .
$$

The analysis by [12] illustrates that a positive upper bound of the system uncertainty is related to the form of the control input vector and has the form

$$
\begin{aligned}
\|\boldsymbol{\rho}(t)\|< & a_{1} a_{2}\|\boldsymbol{u}(t)\|+\left(a_{1} a_{7}+a_{1} d_{1}+a_{1} a_{2} a_{3}\right)+\left(a_{1} a_{8}+a_{1} a_{2} a_{4}\right)\|\boldsymbol{q}\| \\
& +\left(a_{1} a_{9}+a_{1} a_{2} a_{3}\right)\|\dot{\boldsymbol{q}}\|^{2} .
\end{aligned}
$$


Furthermore if the control vector does not contain the acceleration signal $\ddot{\boldsymbol{q}}$ then the system uncertainty will be bounded by a positive function of the position and velocity vector. Assuming the control input is of the form, $\boldsymbol{u}(t)=\boldsymbol{F}(\boldsymbol{q}, \dot{\boldsymbol{q}})$ then there exists a positive function $F_{p}(\boldsymbol{q}, \dot{\boldsymbol{q}})$, such that

$$
\|\boldsymbol{u}(t)\|=\|\boldsymbol{F}(\boldsymbol{q}, \dot{\boldsymbol{q}})\|<F_{p}(\boldsymbol{q}, \dot{\boldsymbol{q}}) .
$$

Using expression (6) in (5) gives: $\|\boldsymbol{\rho}(\boldsymbol{t})\|<F_{d}(\boldsymbol{q}, \dot{\boldsymbol{q}})$, with

$$
\begin{aligned}
F_{d}(\boldsymbol{q}, \dot{\boldsymbol{q}})= & a_{1} a_{2} F_{p}(\boldsymbol{q}, \dot{\boldsymbol{q}})+\left(a_{1} a_{7}+a_{1} d_{1}+a_{1} a_{2} a_{3}\right)+\left(a_{1} a_{8}+a_{1} a_{2} a_{4}\right)\|\boldsymbol{q}\| \\
& +\left(a_{1} a_{9}+a_{1} a_{2} a_{3}\right)\|\dot{\boldsymbol{q}}\|^{2} .
\end{aligned}
$$

The form of this bounded property $\|\boldsymbol{\rho}(t)\|$ has been defined by some researchers as

$$
\|\boldsymbol{\rho}(\boldsymbol{t})\|<b_{o}+b_{1}\|\boldsymbol{q}\|+b_{2}\|\dot{\boldsymbol{q}}\|^{2},
$$

where $b_{o}, b_{1}$ and $b_{2}$ are positive numbers.

\section{Control law calculations}

For clarity, the following analysis includes a greatly reduced and refined summary of that provided by [12] in the basic construction of the controls and Liapunov function. Their notation is adopted. Our theory commences with the incorporation of constraint penalty terms in a chosen Liapunov function, at Equation (14). 
The desired trajectory to be followed by the manipulator may be represented as $\boldsymbol{q}_{r}$ and the output tracking error can be defined as $\boldsymbol{\epsilon}(t)=\boldsymbol{q}-\boldsymbol{q}_{r}$. Ideally we require $\boldsymbol{\epsilon} \rightarrow \mathbf{0}$ resulting in $\boldsymbol{q} \rightarrow \boldsymbol{q}_{r}$. A linearised error system may be obtained from the nominal system described in (4) which is of the form:

$$
\dot{\boldsymbol{e}}=A \boldsymbol{e}+B \boldsymbol{v}, \quad \text { where } \quad A=\left[\begin{array}{ll}
0 & I \\
0 & 0
\end{array}\right], \quad B=\left[\begin{array}{l}
0 \\
I
\end{array}\right],
$$

$\boldsymbol{e}=\left[\begin{array}{ll}\boldsymbol{\epsilon}^{T} & \dot{\boldsymbol{\epsilon}}^{T}\end{array}\right]$ and $\boldsymbol{v}=M_{o}(\boldsymbol{q})^{-1}\left(\boldsymbol{u}_{1}-\boldsymbol{h}_{o}(\boldsymbol{q}, \dot{\boldsymbol{q}})\right)-\ddot{\boldsymbol{q}}_{r}$. A nominal feedback control is used to stabilise the error dynamics of this linearised system, where

$$
\boldsymbol{u}_{1}=\boldsymbol{h}_{o}(\boldsymbol{q}, \dot{\boldsymbol{q}})+M_{o}(\boldsymbol{q})\left(K \boldsymbol{e}+\ddot{\boldsymbol{q}}_{r}\right)
$$

where $K=\left[\begin{array}{ll}-K_{1} & -K_{2}\end{array}\right]$, and matrix $K$ is designed such that $A_{1}=A+$ $B K$. The control input will have the form

$$
\boldsymbol{u}(t)=\boldsymbol{u}_{1}+\boldsymbol{u}_{o}
$$

where $\boldsymbol{u}_{1}$ is designed for the nominal system in (4) and $\boldsymbol{u}_{o}$ is a compensator used to eliminate the effects of system uncertainty in (5). By using Equations (3), (4), (9) and (10) the error dynamic of the system with uncertain dynamics is

$$
\dot{\boldsymbol{e}}=A_{1} \boldsymbol{e}+B M_{o}(\boldsymbol{q})^{-1} \boldsymbol{u}_{o}+B M_{o}(\boldsymbol{q})^{-1} \boldsymbol{\rho}(t) .
$$

The compensator $\boldsymbol{u}_{o}$ is designed using a sliding mode technique. The purpose of designing it in this way is to obtain a finite time error convergence. Furthermore an adaptive mechanism is employed to adaptively estimate the 
parameters of the upper bound of the system uncertainty $\boldsymbol{\rho}(t)$. Estimates of $b_{o}, b_{1}$ and $b_{2}$ in (8) are defined as $\hat{b}_{o}, \hat{b}_{1}$ and $\hat{b}_{2}$ and a parameter error estimate is defined as $\boldsymbol{\psi}(t)=\hat{\boldsymbol{b}}-\boldsymbol{b}$. The system uncertainty are updated by the following adaptive laws:

$$
\begin{aligned}
& \hat{\dot{b}_{o}}=x_{o}\left\|C_{2} M_{o}(\boldsymbol{q})^{-1}\right\|\|\boldsymbol{S}\|, \\
& \hat{\dot{b}_{1}}=x_{1}\left\|C_{2} M_{o}(\boldsymbol{q})^{-1}\right\|\|\boldsymbol{S}\|\|\boldsymbol{q}\|, \\
& \hat{b_{2}}=x_{2}\left\|C_{2} M_{o}(\boldsymbol{q})^{-1}\right\|\|\boldsymbol{S}\|\|\boldsymbol{q}\|\|\boldsymbol{q}\|^{2}
\end{aligned}
$$

where $x_{i}(i=0,1,2)$ are arbitrary positive numbers and $\hat{b}_{i}(i=0,1,2)$ have arbitrary positive initial values. As [12] highlights, the form of Equation (13), illustrates that the system uncertainty are estimated in the Liapunov sense. Consequently it is not necessary for the estimates to converge to their true values but instead the values of the estimates are adaptively adjusted until the terminal sliding variable vector $\boldsymbol{S}$ converges to zero. Once this convergence has been reached the estimates become constants so as to retain the error dynamics on the terminal sliding mode. This is important in order for a finite error convergence to be achieved on the terminal sliding mode.

To design the compensator $\boldsymbol{u}_{o}$ in (11) we first define the MIMO terminal sliding variable vector $\boldsymbol{S}=C \tilde{\boldsymbol{e}}$, where

$$
C=\left[\begin{array}{ll}
C_{1} & C_{2}
\end{array}\right]=\left[\begin{array}{ccc|ccc}
c_{11} & & 0 & 1 & & 0 \\
& \ddots & & & \ddots & \\
0 & & c_{n n} & 0 & & 1
\end{array}\right]
$$


$c_{i i}>0(i=1, \ldots, n)$ and $\tilde{\boldsymbol{\epsilon}}=\left[\epsilon_{1}^{p_{1} / p_{2}} \cdots \epsilon_{n}^{p_{1} / p_{2}} \dot{\epsilon}_{1} \cdots \dot{\epsilon}_{n}\right]^{T}$. Matrix $C_{2}$ is chosen as the identity matrix to simplify controller design. However a different form may be employed to improve the convergence of the error dynamics in the terminal sliding mode. The selection of $p_{1}$ and $p_{2}$ is critical in the design of an appropriate sliding mode control system. Generally $p_{1}$ and $p_{2}$ are positive odd integers which satisfy the conditions $p_{2}>p_{1}$ and $p_{2}=(2 m+1)$, for $m=1,2, \ldots$. Indeed it is important that $p_{1} / p_{2}<1$ so that the tracking errors, $\epsilon_{i}$, converge to zero in a finite time. Note that $\tilde{\boldsymbol{e}}$ can be expressed as $\tilde{\boldsymbol{e}}=\boldsymbol{e}+\Delta \tilde{\boldsymbol{e}}$, where $\Delta \tilde{\boldsymbol{e}}=\left[\begin{array}{lllllll}\epsilon_{1}^{p_{1} / p_{2}}-\epsilon_{1} & \cdots & \epsilon_{n}^{p_{1} / p_{2}}-\epsilon_{n} & 0 & \cdots & 0\end{array}\right]^{T}$. This means that the MIMO terminal sliding mode variable vector, $\boldsymbol{S}$, has the form

$$
\begin{aligned}
\boldsymbol{S} & =C \tilde{\boldsymbol{e}} \\
& =C(\boldsymbol{e}+\Delta \tilde{\boldsymbol{e}}) \\
& =C \boldsymbol{e}+C_{1}(\tilde{\boldsymbol{\epsilon}}-\boldsymbol{\epsilon}),
\end{aligned}
$$

where $\tilde{\boldsymbol{e}}=\left[\begin{array}{lll}\epsilon_{1}^{p_{1} / p_{2}} & \cdots & \epsilon_{n}^{p_{1} / p_{2}}\end{array}\right]^{T}$. The convenience of redefining $\boldsymbol{S}$ in this way is that controller design and stability analysis is made easier because this redefinition highlights the relationship between the sliding mode variable vector and the error dynamics of the robot control system.

The Liapunov function defined by the authors in [12] is

$$
V=\frac{1}{2}\left(\boldsymbol{S}^{T} \boldsymbol{S}+\sum_{i=0}^{2} x_{i}^{-1} \hat{b}_{i}^{2}\right),
$$

where $\tilde{b}_{i}=b_{i}-\hat{b}_{i}, \dot{\tilde{b}}_{i}=-\dot{\hat{b}}_{i}$. We incorporate $m$ physical constraints into the 
system by defining penalty functions, $V_{i}$, required to be greater than zero and associated positive constants $\gamma_{i}(i=1, \ldots, m)$.

These constraints are incorporated as multipliers in a new Liapunov function defined as

$$
V=\frac{1}{2}\left(\boldsymbol{S}^{T} \boldsymbol{S}+\sum_{i=0}^{2} x_{i}^{-1} \hat{b}_{i}^{2}\right) \sum_{i=1}^{m} \frac{\gamma_{i}}{V_{i}}=\frac{1}{2}\left(\boldsymbol{S}^{T} \boldsymbol{S}+\sum_{i=0}^{2} x_{i}^{-1} \hat{b}_{i}^{2}\right) A,
$$

where $A$ encapsulates all the incorporated constraints

$$
A=\sum_{i=1}^{m} \frac{\gamma_{i}}{V_{i}}
$$

By considering the error dynamics of (12) for the robotic manipulator system in (2) and defining the new compensator $\boldsymbol{u}_{o}$, which incorporates constraint satisfaction as

$$
\boldsymbol{u}_{o}= \begin{cases}\frac{\left(\boldsymbol{S}^{T} C_{2} M_{o}(\boldsymbol{q})^{-1}\right)^{T}}{A\left\|\boldsymbol{S}^{T} C_{2} M_{o}(\boldsymbol{q})^{-1}\right\|^{2}} w, & \|\boldsymbol{S}\| \neq 0 \text { with } \epsilon_{i} \neq 0, \\ \mathbf{0}, & \|\boldsymbol{S}\|=0 \text { or }\|\boldsymbol{S}\| \neq 0 \text { with } \epsilon_{i}=0\end{cases}
$$

where

$$
\begin{aligned}
w= & -\boldsymbol{S}^{T} C A\left(A_{1} \boldsymbol{e}-\frac{B \boldsymbol{e}}{A}\right)-A\|\boldsymbol{S}\|\left\|C_{2} M_{o}(\boldsymbol{q})^{-1}\right\|\left(\hat{b}_{o}+\hat{b}_{1}\|\boldsymbol{q}\|+\hat{b}_{2}\|\dot{\boldsymbol{q}}\|^{2}\right) \\
& -\boldsymbol{S}^{T} C_{1} A\left(\left(\boldsymbol{\epsilon}_{r}-\dot{\boldsymbol{\epsilon}}\right)-(\tilde{\boldsymbol{\epsilon}}-\boldsymbol{\epsilon}) \frac{B}{A}\right)
\end{aligned}
$$


and

$$
\boldsymbol{\epsilon}_{r}=\operatorname{diag}\left(\frac{p_{1}}{p_{2}} \epsilon_{1}^{p_{1} /\left(p_{2}-1\right)}, \ldots, \frac{p_{1}}{p_{2}} \epsilon_{n}^{p_{1} /\left(p_{2}-1\right)}\right) \dot{\boldsymbol{\epsilon}}_{r}, \quad B=\frac{1}{2} \sum_{i=1}^{m} \frac{\gamma_{i} \dot{V}_{i}}{V_{i}^{2}}
$$

and $p_{2}>p_{1} \geq\left(p_{2}+1\right) / 2$. Differentiating Equation (14) with respect to time we obtain

$$
\begin{aligned}
\dot{V}= & \left(\boldsymbol{S}^{T} \dot{\boldsymbol{S}}+\sum_{i=0}^{2} x_{i}^{-1} \hat{b}_{i} \hat{\hat{b}}_{i}\right) A+\frac{1}{2}\left(\boldsymbol{S}^{T} \boldsymbol{S}+\sum_{i=0}^{2} x_{i}^{-1} \hat{b}_{i}^{2}\right) B \\
= & \boldsymbol{S}^{T}(\dot{\boldsymbol{S}} A-\boldsymbol{S} B)-\left[\left(\sum_{i=0}^{2} x_{i}^{-1} \hat{b}_{i} \hat{\hat{b}}_{i}\right) A+\left(\boldsymbol{S}^{T} \boldsymbol{S}+\sum_{i=0}^{2} x_{i}^{-1} \hat{b}_{i}^{2}\right) B\right] \\
= & \boldsymbol{S}^{T}\left[C A_{1} \boldsymbol{e}+C B M_{o}(\boldsymbol{q})^{-1} \boldsymbol{\rho}(t)+c_{1} \boldsymbol{\epsilon}_{r}-c_{1} \dot{\boldsymbol{\epsilon}}-\boldsymbol{S} B\right]-Z \\
= & \boldsymbol{S}^{T} A C A_{1} \boldsymbol{e}+\boldsymbol{S}^{T} A C_{2} M_{o}(\boldsymbol{q})^{-1} \boldsymbol{u}_{o}+\boldsymbol{S}^{T} A C_{2} M_{o}(\boldsymbol{q})^{-1} \boldsymbol{\rho}(t)+\boldsymbol{S}^{T} A c_{1} \boldsymbol{\epsilon}_{r} \\
& -\boldsymbol{S}^{T} c_{1} \dot{\boldsymbol{\epsilon}}-\boldsymbol{S}^{T} B C \boldsymbol{e}-\boldsymbol{S}^{T} B c_{1}(\tilde{\boldsymbol{\epsilon}}-\boldsymbol{\epsilon})-Z \\
= & \boldsymbol{S}^{T} C\left(A_{1} \boldsymbol{e} A-B \boldsymbol{e}\right)+\boldsymbol{S}^{T} C_{2} M_{o}(\boldsymbol{q})^{-1} \boldsymbol{u}_{o} A+\boldsymbol{S}^{T} C_{2} M_{o}(\boldsymbol{q})^{-1} \boldsymbol{\rho}(t) A \\
& +\boldsymbol{S}^{T} c_{1}\left[\left(\boldsymbol{\epsilon}_{r}-\dot{\boldsymbol{\epsilon}}\right)-(\tilde{\boldsymbol{\epsilon}}-\boldsymbol{\epsilon}) B\right]-Z \\
= & \boldsymbol{S}^{T} C\left(A_{1} \boldsymbol{e} A-B \boldsymbol{e}\right)+\boldsymbol{S}^{T} C_{2} M_{o}(\boldsymbol{q})^{-1} \boldsymbol{u}_{o} A+\boldsymbol{S}^{T} C_{2} M_{o}(\boldsymbol{q})^{-1} \boldsymbol{\rho}(t) A \\
& +\boldsymbol{S}^{T} c_{1} A\left[\left(\boldsymbol{\epsilon}_{r}-\dot{\boldsymbol{\epsilon}}\right)-(\tilde{\boldsymbol{\epsilon}}-\boldsymbol{\epsilon}) \frac{B}{A}\right]-Z \\
= & \boldsymbol{S}^{T} C\left(A_{1} \boldsymbol{e} A-B \boldsymbol{e}\right)+w+\boldsymbol{S}^{T} C_{2} M_{o}(\boldsymbol{q})^{-1} \boldsymbol{\rho}(t) A \\
& +\boldsymbol{S}^{T} c_{1} A\left[(\boldsymbol{\epsilon}-\dot{\boldsymbol{\epsilon}})-(\tilde{\boldsymbol{\epsilon}}-\boldsymbol{\epsilon}) \frac{B}{A}\right]-Z \\
= & -A\|\boldsymbol{S}\|\left\|C_{2} M_{o}(\boldsymbol{q})^{-1}\right\|\left(\hat{b}_{o}+\hat{b}_{1}\|\boldsymbol{q}\|+\hat{b}_{2}\|\dot{\boldsymbol{q}}\|^{2}\right) A
\end{aligned}
$$




$$
\begin{aligned}
& =\boldsymbol{S}^{T} C_{2} M_{o}(\boldsymbol{q})^{-1} \boldsymbol{\rho}(t) A-\|\boldsymbol{S}\|\left\|C_{2} M_{o}(\boldsymbol{q})^{-1}\right\|\left(\hat{b}_{o}+\hat{b}_{1}\|\boldsymbol{q}\|+\hat{b}_{2}\|\dot{\boldsymbol{q}}\|^{2}\right) A \\
& \leq-A\left\|C_{2} M_{o}(\boldsymbol{q})^{-1}\right\|\left[\left(\hat{b}_{o}+\hat{b}_{1}\|\boldsymbol{q}\|+\hat{b}_{2}\right)-\|\boldsymbol{\rho}(t)\|\right]\|\boldsymbol{S}\| \\
& =-\eta\|\boldsymbol{S}\|<0
\end{aligned}
$$

where $\eta=A\left\|C_{2} M_{o}(\boldsymbol{q})^{-1}\right\|\left[\left(\hat{b}_{o}+\hat{b}_{1}\|\boldsymbol{q}\|+\hat{b}_{2}\right)-\|\boldsymbol{\rho}(\boldsymbol{t})\|\right]$ and

$$
\begin{aligned}
Z= & -A\left[\sum_{i=0}^{2} x_{i}^{-1} \hat{b}_{i}^{2} \hat{b}_{i}+\frac{B \tilde{b}_{i}}{A}\right] \\
= & -A\|\boldsymbol{S}\|\left\|C_{2} M_{o}(\boldsymbol{q})^{-1}\right\|\left[\left(\hat{b}_{o}+\hat{b}_{1}\|\boldsymbol{q}\|+\hat{b}_{2}\|\dot{\boldsymbol{q}}\|^{2}\right)\right. \\
& \left.-\left(b_{o}+b_{1}\|\boldsymbol{q}\|+b_{2}\|\dot{\boldsymbol{q}}\|^{2}\right)\right] .
\end{aligned}
$$

Since $\eta>0$, Equation (16) indicates that the terminal sliding variable vector $\boldsymbol{S}$ converges to zero in a finite time.

\section{Applications to a 2 link manipulator}

Our previous theory has been developed for a general $n$-joint rigid robotic manipulator. We shall examine its application to a two link robotic manipulator, it being the same example as given in [12], to enable the reader to make comparison with their results.

The dynamic equations of the two link robotic manipulator are expressed in state variable form as $x_{1}=q_{1}, x_{2}=\dot{q}_{1}, x_{3}=q_{2}, x_{4}=\dot{q}_{2}$ with $\boldsymbol{x}=$ 
$\left[\begin{array}{llll}x_{1} & x_{2} & x_{3} & x_{4}\end{array}\right]^{T}$. The dynamics of this specific system is given by the equations

$$
\begin{aligned}
\dot{x}_{1}= & x_{2}, \\
\dot{x}_{2}= & \frac{1}{a_{11}}\left[\beta_{12} \dot{q}_{1}\left(\dot{q}_{1}+\dot{q}_{2}\right)\left(1+\frac{a_{12}^{2}}{\left(a_{11} a_{12}-a_{12}^{2}\right)}\right)+\gamma_{1} g+u_{1}\right. \\
& \left.\quad-\frac{a_{12}}{\left(a_{11} a_{12}-a_{12}^{2}\right)}\left(a_{11}\left(\gamma_{2} g-\beta_{12} \dot{q}_{2}+u_{2}\right)-a_{12}\left(\gamma_{1} g+u_{2}\right)\right)\right], \\
\dot{x}_{3}= & x_{4}, \quad \frac{1}{\dot{x}_{4}=} \frac{1}{\left(a_{11} a_{12}-a_{12}^{2}\right)}\left[a_{11}\left(\gamma_{2} g-\beta_{12} \dot{q}_{2}^{2}+u_{2}\right)\right. \\
& \left.\quad-a_{12}\left(\beta_{12} \dot{q}_{1}\left(\dot{q}_{1}+\dot{q}_{2}\right)+\gamma_{1} g+u_{2}\right)\right],
\end{aligned}
$$

where

$$
\begin{aligned}
a_{11} & =\left(m_{1}+m_{2}\right) r_{1}^{2}+m_{2} r_{2}^{2}+2 m_{2} r_{1} r_{2} \cos \left(q_{2}\right)+J_{1}, \\
a_{12} & =m_{2} r_{2}^{2}+2 m_{2} r_{1} r_{2} \cos \left(q_{2}\right), \\
a_{22} & =m_{2} r_{2}^{2}+J_{2}, \\
\beta_{12} & =m_{2} r_{1} r_{2} \sin \left(q_{2}\right), \\
\gamma_{1}\left(q_{1}, q_{2}\right) & =-\left(\left(m_{1}+m_{2}\right) r_{1} \cos \left(q_{2}\right)+m_{2} r_{2} \cos \left(q_{1}+q_{2}\right)\right), \\
\gamma_{2}\left(q_{1}, q_{2}\right) & \left.=-m_{2} r_{2} \cos \left(q_{1}+q_{2}\right)\right) .
\end{aligned}
$$

The parameter values are

$$
\begin{aligned}
r_{1} & =1.0 \mathrm{~m}, \quad r_{2}=0.8 \mathrm{~m} \\
J_{1} & =5 \mathrm{~kg} \mathrm{~m}, \quad J_{2}=5 \mathrm{~kg} \mathrm{~m}, \\
m_{1} & =0.5 \mathrm{~kg}, \quad m_{2}=1.5 \mathrm{~kg} .
\end{aligned}
$$


We impose the constraints $0<x_{1}<0.85$ and $0<x_{3}<1.571$ on the system variables. This upper limit of 0.85 was chosen as $x_{1}$ measures the angular movement of link one and this link corresponds in a physical sense to a human upper arm; between the elbow and the shoulder. Such an angular restriction on $x_{1}$ would realistically reflect a possible range of movement of this human limb. The lower boundaries on $q_{1}$ and $q_{2}$ are set to 0 radians and the upper boundaries are set to 0.85 and 1.571 radians respectively.

In [12] the desired trajectory reference signals were defined as

$$
\begin{aligned}
q_{r 1} & =1.25-\frac{7}{5} e^{-t}+\frac{7}{20} e^{-4 t}, \\
\text { and } \quad q_{r 2} & =1.25+e^{-t}+-\frac{1}{4} e^{-4 t}
\end{aligned}
$$

We have modified the desired trajectory reference signals as:

$$
\begin{aligned}
q_{r 1} & =0.85-\frac{7}{5} e^{-t}+\frac{7}{20} e^{-4 t}, \\
\text { and } q_{r 2} & =1.25+e^{-t}+-\frac{1}{4} e^{-4 t}
\end{aligned}
$$

This has been done since the steady state of 1.25 in $q_{r 1}$ lies outside the constraint boundaries of $x_{1}$.

The desired error dynamics of the closed loop system, as defined by [12], is $\ddot{\epsilon}_{k}+5 \dot{\epsilon}_{k}+4 \epsilon_{k}=0$, where $k=1,2,3,4$. The feedback matrix $K$ is designed 
as

$$
K=\left[\begin{array}{ll}
-K_{1} & -K_{2}
\end{array}\right]=\left[\begin{array}{cccc}
-k_{1} & 0 & -k_{2} & 0 \\
0 & -k_{1} & 0 & -k_{2}
\end{array}\right]=\left[\begin{array}{cccc}
-4 & 0 & -5 & 0 \\
0 & -4 & 0 & -5
\end{array}\right]
$$

The terminal sliding mode is defined as:

$$
\epsilon_{1}^{3 / 5}+\dot{\epsilon}_{1}=0, \text { and } \epsilon_{2}^{3 / 5}+\dot{\epsilon}_{2}=0 .
$$

The physical constraints are now incorporated into the system by defining penalty functions

$$
\begin{aligned}
V_{1} & =x_{1}, \\
V_{2} & =0.85-x_{1}, \\
V_{3} & =x_{3}, \\
\text { and } \quad V_{4} & =1.571-x_{3} .
\end{aligned}
$$

The function $V_{2}$, which corresponds to the constraint on variable $x_{1}$, is required to be strictly positive, that is, $V_{2}>0$. Similarly we require $V_{1}>0$, $V_{3}>0$ and $V_{4}>0$. The nominal values of $m_{1}$ and $m_{2}$ are

$$
\hat{m}_{1}=0.4 \mathrm{~kg} \quad \text { and } \quad \hat{m}_{2}=1.2 \mathrm{~kg} \text {. }
$$

As in [12] at $t=4$ seconds the manipulator picks up a 0.5 kilogram object. Consequently the mass of link 2 is increased from $1.5 \mathrm{~kg}$ to $2.0 \mathrm{~kg}$. Other system parameters are assumed known. The nominal system is then constructed from the known system dynamics. 
We now consider the expansion of Equation (10) in order to show the full form of the controls used:

$\boldsymbol{u}_{1}=\left[\begin{array}{cc}\beta_{12} \dot{\boldsymbol{q}}_{1}\left(\dot{\boldsymbol{q}}_{1}+2 \dot{\boldsymbol{q}}_{2}\right)+\gamma_{1} \boldsymbol{g}+a_{11}\left(-k_{1} \epsilon_{1}-k_{2} \dot{\epsilon}_{1}+\ddot{\boldsymbol{q}}_{r 1}\right)+a_{12}\left(-k_{1} \epsilon_{2}-k_{2} \dot{\epsilon}_{2}+\ddot{\boldsymbol{q}}_{r 2}\right) \\ -\beta_{12} \dot{\boldsymbol{q}}_{2}^{2}+\gamma_{2} \boldsymbol{g}+a_{12}\left(-k_{1} \epsilon_{1}-k_{2} \dot{\epsilon}_{1}+\ddot{\boldsymbol{q}}_{r 1}\right)+a_{22}\left(-k_{1} \epsilon_{2}-k_{2} \dot{\epsilon}_{2}+\ddot{\boldsymbol{q}}_{r 2}\right)\end{array}\right]$

\section{Simulation discussion}

We report here on four simulations. In all the state equations were integrated using a Runge-Kutta algorithm with step size $\delta t=0.01$. Simulations 5.1, 5.2 and 5.3 highlight the convergence to a steady state value whereas simulation 5.4 demonstrates that the model will also track an oscillating reference signal. Henceforth we adopt a non-dimensionalised unit of time. All control outputs exhibit a large amount of chattering whose extent is difficult to discern over the full 20 time units. In order to best highlight the extent of chattering we have restricted our figures illustrating control outputs to the first 5 time unit period. The control response over the remainder of the time unit exhibits a similar chattering response. 


\subsection{Simulation A: tracking a fixed point reference sig- nal}

This simulation highlights the operation of the manipulator when tracking to a steady state value; $q_{1}$ and $q_{1}$ converge to 0.85 and 1.25 respectively. Here the manipulator is initially centrally located in the workspace defined by the boundary conditions placed upon $q_{1}$ and $q_{2}$. The initial angular positions of $q_{1}$ and $q_{2}$ are set as 0.8 and their initial angular velocities are set at 0.0 . Investigations of the resulting control magnitudes and parameter assignment are conducted to ascertain how well convergence is achieved when the manipulator is operating in its boundary regions as outlined and to compare the results obtained here with those obtained by [12].

The initial state values of the system were selected as

$$
\left[q_{1}(0) q_{2}(0)\right]^{T}=\left[\begin{array}{ll}
0.8 & 0.8
\end{array}\right]^{T} \text { and }\left[\dot{q}_{1}(0) \dot{q}_{2}(0)\right]^{T}=\left[\begin{array}{ll}
0 & 0
\end{array}\right]^{T} \text {. }
$$

This differs slightly from the initial values of the system set by [12]:

$$
\left[q_{1}(0) q_{2}(0)\right]^{T}=\left[\begin{array}{lll}
0.8 & 1.8
\end{array}\right]^{T} \text { and }\left[\dot{q}_{1}(0) \dot{q}_{2}(0)\right]^{T}=\left[\begin{array}{ll}
0 & 0
\end{array}\right]^{T} \text {. }
$$

The reason for the difference in our initial values and [12] arises from the upper limit we have imposed upon $q_{2}$. The initial parameter values and the initial parameter estimates were set as:

$$
\hat{\boldsymbol{b}}=\left[\begin{array}{lll}
b_{o} & b_{1} & b_{2}
\end{array}\right]^{T}=\left[\begin{array}{llll}
1 & 1 & 1 & 1
\end{array}\right]^{T} \quad \text { and } \quad \boldsymbol{b}=\left[\begin{array}{lll}
\hat{b}_{o} & \hat{b}_{1} & \hat{b}_{2}
\end{array}\right]^{T}=\left[\begin{array}{llll}
1 & 1 & 1 & 1
\end{array}\right]^{T} .
$$


The avoidance parameters adopted were

$$
\gamma_{1}=\gamma_{2}=\gamma_{3}=\gamma_{4}=1
$$

This simulation resulted in:

\begin{tabular}{ll}
\hline Final Time: & 20 time periods \\
Final Parameter Estimates: & $\hat{\boldsymbol{b}}=\left[\begin{array}{lll}\hat{b}_{0} & \hat{b}_{1} & \hat{b}_{2}\end{array}\right]^{T}=\left[\begin{array}{lll}1.44 & 1.52 & 1.03\end{array}\right]^{T}$ \\
Controls: & $\min =-23.329$ and $\max =15.935$ \\
Convergence: & $t_{1}=5.63$ and $t_{2}=5.30$ \\
\hline
\end{tabular}

See in Figure 1 that the close tracking of $q_{1}$ to the desired path of $q_{r 1}$ and the close tracking of $q_{3}$ to the desired path of $q_{r 2}$, while initially somewhat offset, both occur quite quickly; $t_{1}$ and $t_{2}$ respectively. As highlighted in Figures 2, 3 and 4, in order to achieve close tracking in either case, quite moderately sized controls are required. Notice that a large amount of chattering is also evident. It is prevalent in $u_{2}$ to a greater extent. Since it is not easy to discern the extent to which the chattering is occurring we have included Figure 2 which illustrates the form of the control over the whole 20 time units and as a contrast we have included Figure 3 which better illustrates the extent of chattering over the first period of 5 time units. The control response over the remainder of the time unit would exhibit a similar chattering response.

These results correlate well with the results of [12]: 


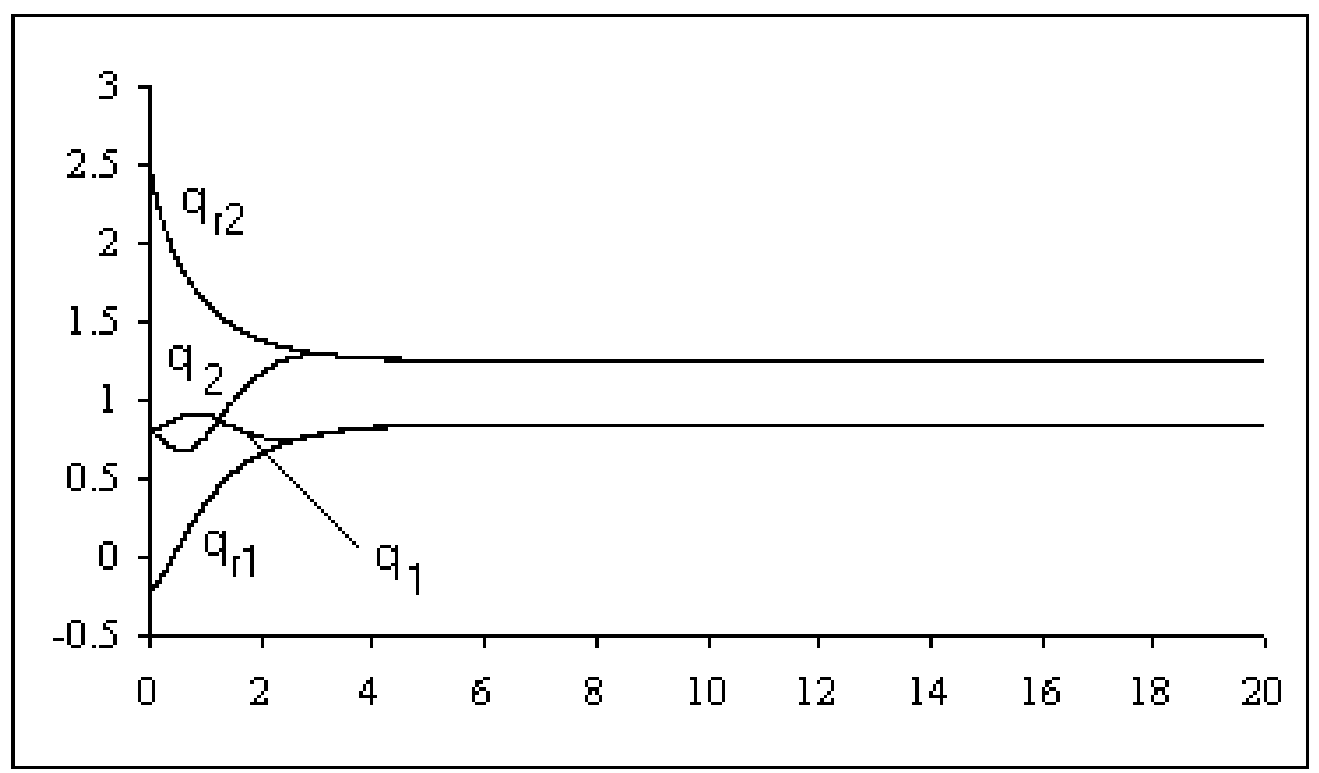

FiguRE 1: Simulation 5.1 Trajectories $q_{1}$ tracking $q_{r 1}$ and $q_{3}$ tracking $q_{r 2}$ 


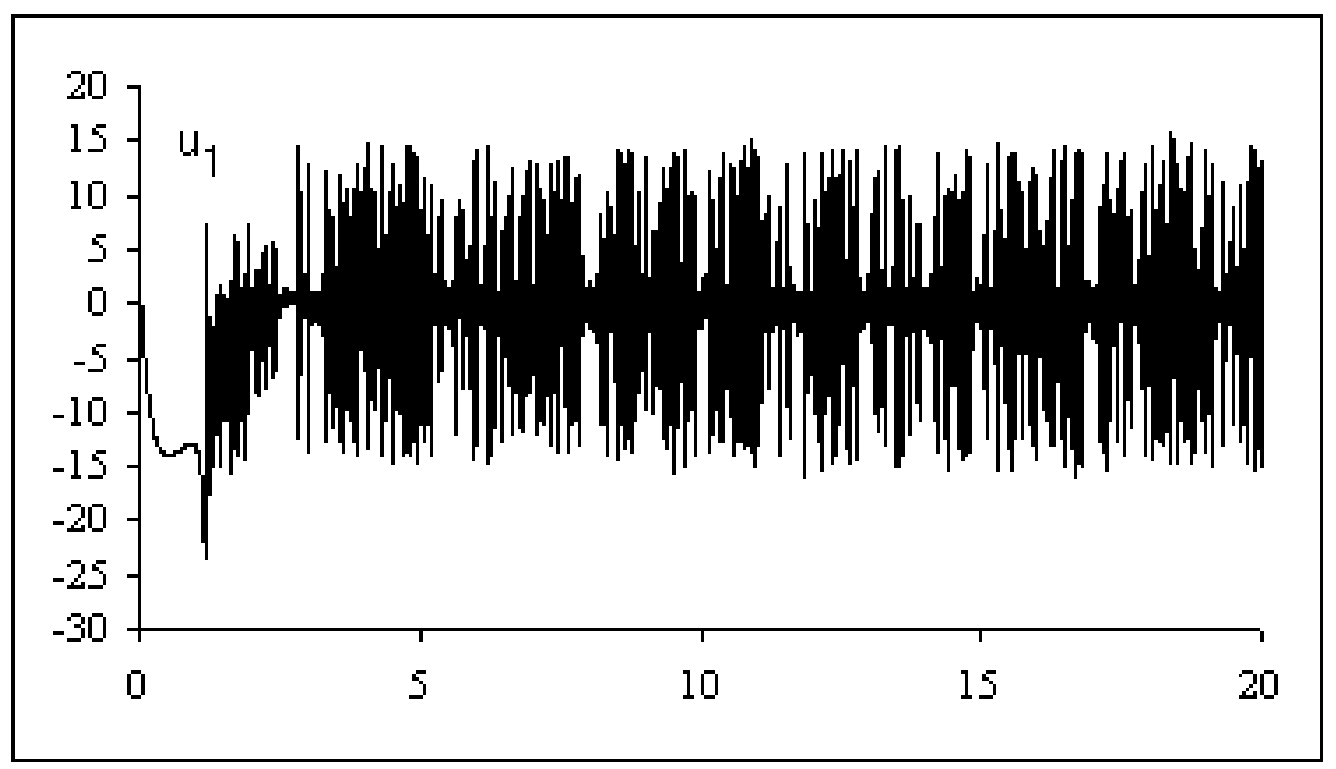

Figure 2: Simulation 5.1 control $u_{1}$ for 20 time units 


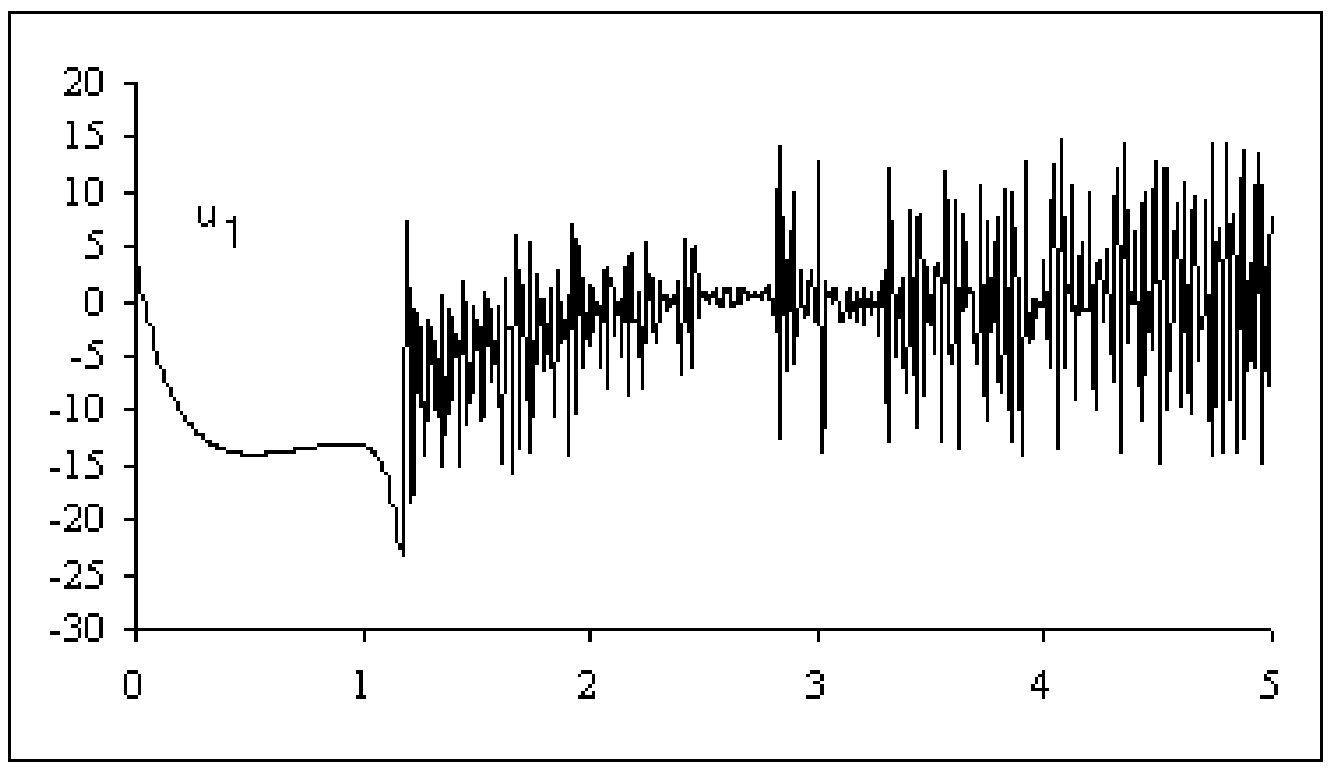

Figure 3: Simulation 5.1 control $u_{1}$ for 5 time units 


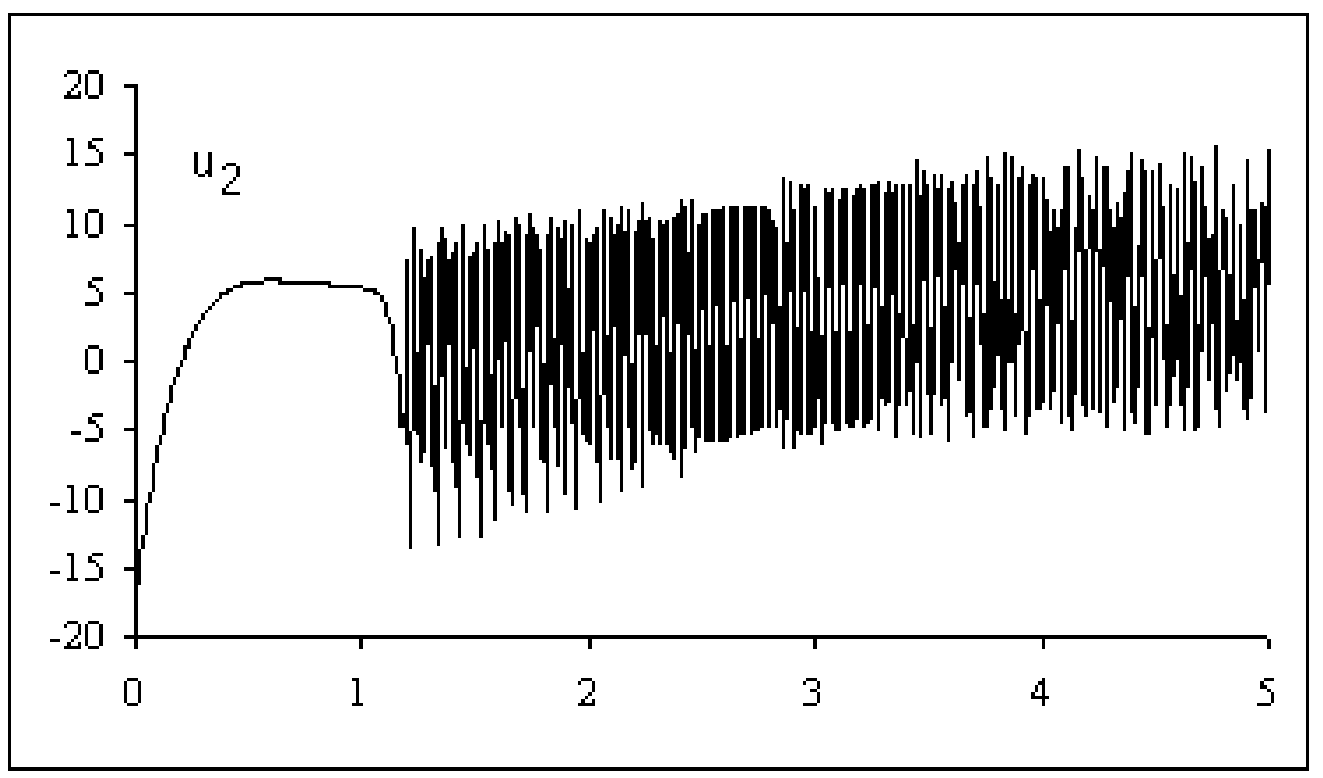

Figure 4: Simulation 5.1 control $u_{2}$ for 5 time units 


\begin{tabular}{ll}
\hline Final Parameter Estimates: & $\hat{\boldsymbol{b}}=\left[\begin{array}{lll}\hat{b}_{0} & \hat{b}_{1} \hat{b}_{2}\end{array}\right]^{T}=\left[\begin{array}{lll}10.50 & 1.50 & 0.50\end{array}\right]^{T}$ \\
Controls: & $\min =-32$ and $\max =22$ \\
Convergence: & $t_{1}=1.8$ and $t_{2}=1.1$ \\
\hline
\end{tabular}

Even though our simulation takes longer to reach convergence we require a much lower range in the size of the controls required to obtain convergence for [12].

Zhihong [12] does not provide graphical plots of the tracking error. From our simulation results the rapid rate of convergence of both tracking errors to zero, as shown in Figure 5, validates the swiftness in which the close tracking of the desired paths, highlighted by Figure 1, occurs.

As outlined in the control law calculation section it is not necessary for the estimates to converge to zero. In fact the values of the estimates are adaptively adjusted until the terminal sliding variable vector $S$ converges to zero. Once terminal sliding variable convergence has been achieved the estimates converge to a constant value. In Figure 6 parameter estimate $\hat{b}_{2}$ shows an early convergence to a constant value of 0.44 and interpreting this means that from an initial value of 1.0 , the parameter estimate $\hat{b}_{2} \rightarrow 1.44$. While $\hat{b}_{0}$ and $\hat{b}_{1}$, as shown by Figure 6 , also converge to a constant value, 0.52 and 0.03 respectively. This means that from an initial value of 1.0 , the parameter estimates $\hat{b}_{0}$ and $\hat{b}_{1}$ converge to 1.52 and 1.03 respectively. The controls are evauluated, keeping all other parameters the same as previously and adopting the avoidance parameters:

$$
\gamma_{1}=10, \quad \gamma_{2}=80, \quad \gamma_{3}=50, \quad \gamma_{4}=5
$$




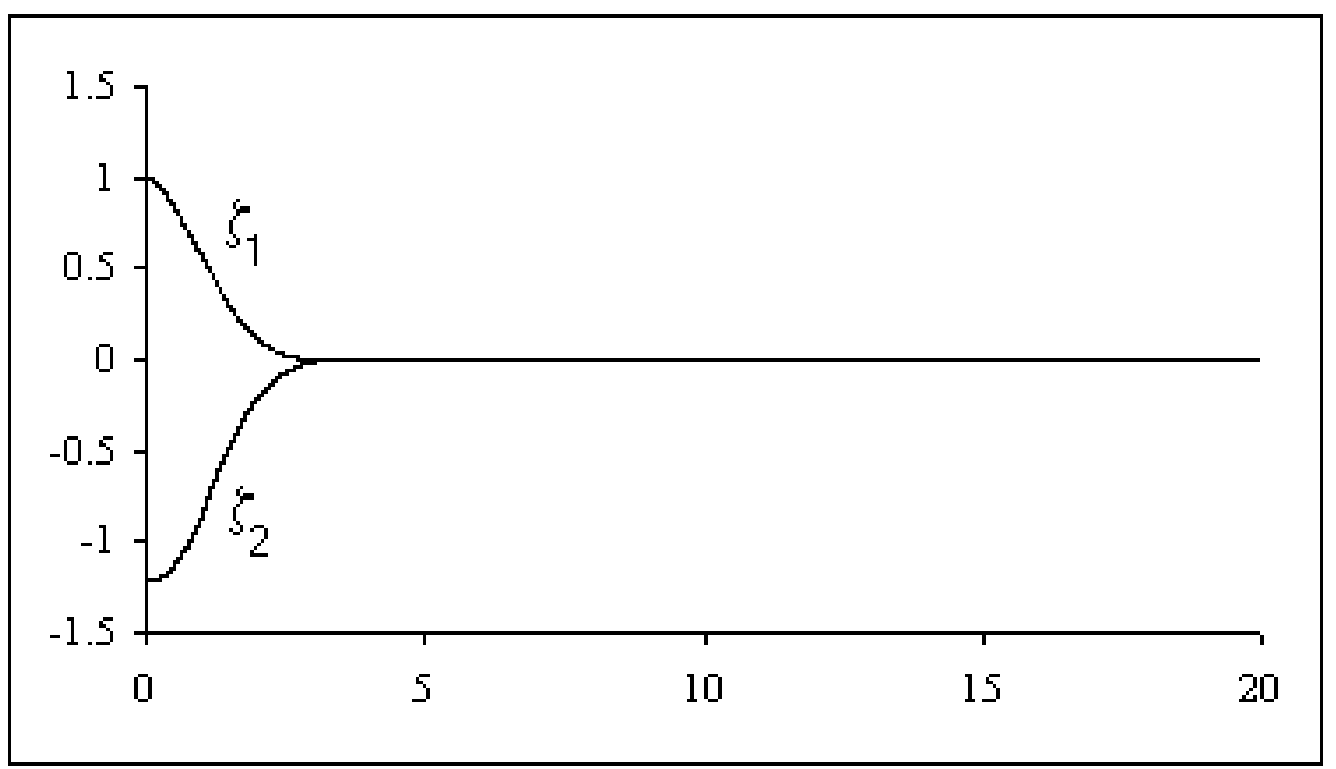

Figure 5: Simulation 5.1 Tracking errors $\epsilon_{1}$ and $\epsilon_{2}$ 


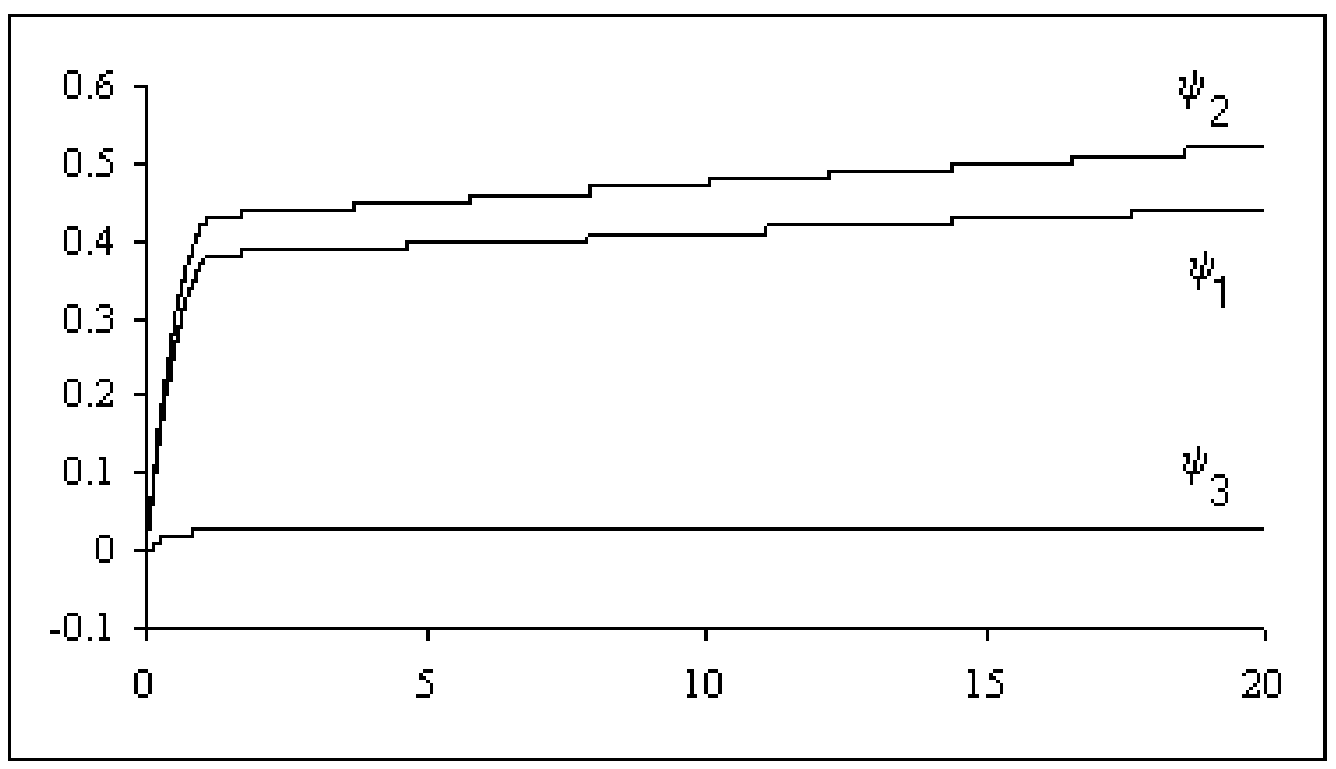

Figure 6: Simulation 5.1 Parameter error estimates $\psi_{1}, \psi_{2}, \psi_{3}$ 
This simulation resulted in:

\begin{tabular}{ll}
\hline Final Time: & 20 time periods \\
Final Parameter Estimates: & $\hat{\boldsymbol{b}}=\left[\begin{array}{lll}\hat{b}_{0} & \hat{b}_{1} & \hat{b}_{2}\end{array}\right]^{T}=\left[\begin{array}{lll}1.41 & 1.49 & 1.02\end{array}\right]^{T}$ \\
Controls: & $\min =-22.335$ and $\max =15.661$ \\
Convergence: & $t_{1}=5.63$ and $t_{2}=5.29$ \\
\hline
\end{tabular}

\subsection{Simulation B: tracking a fixed point reference sig- nal}

This simulation again highlights the operation of the manipulator when tracking to a steady state value; $q_{1}$ and $q_{1}$ converge to 0.85 and 1.25 respectively but the initial angular position of $q_{2}$ is set close to its upper boundary and is moving toward this upper boundary with an initial angular velocity of 0.1 while its initial angular position of $q_{1}$ is set close to its lower boundary and is moving toward this lower boundary with an initial angular velocity of -0.3 . Investigations of the resulting control magnitudes and parameter assignment are conducted to ascertain how well convergence is achieved when the manipulator is operating in its boundary regions as outlined.

The initial state values of the system were selected as:

$$
\left[q_{1}(0) q_{2}(0)\right]^{T}=\left[\begin{array}{lll}
0.10 & 1.56
\end{array}\right]^{T} \quad \text { and } \quad\left[\dot{q}_{1}(0) \dot{q}_{2}(0)\right]^{T}=\left[\begin{array}{lll}
-0.3 & 0.1
\end{array}\right]^{T}
$$


The initial parameter values and the initial parameter estimates were set as:

$$
\hat{\boldsymbol{b}}=\left[\begin{array}{lll}
b_{o} & b_{1} & b_{2}
\end{array}\right]^{T}=\left[\begin{array}{llll}
1 & 1 & 1 & 1
\end{array}\right]^{T} \quad \text { and } \quad \boldsymbol{b}=\left[\begin{array}{lll}
\hat{b}_{o} & \hat{b}_{1} & \hat{b}_{2}
\end{array}\right]^{T}=\left[\begin{array}{llll}
1 & 1 & 1 & 1
\end{array}\right]^{T} .
$$

The avoidance parameters adopted were:

$$
\gamma_{1}=1, \quad \gamma_{2}=1, \quad \gamma_{3}=1000, \quad \gamma_{4}=1
$$

This simulation resulted in:

\begin{tabular}{ll}
\hline Final Time: & 20 time periods \\
Final Parameter Estimates: & $\hat{\boldsymbol{b}}=\left[\begin{array}{lll}\hat{b}_{0} & \hat{b}_{1} & \hat{b}_{2}\end{array}\right]^{T}=\left[\begin{array}{lll}1.13 & 1.20 & 1.01\end{array}\right]^{T}$ \\
Controls: & $\min =-12.459$ and $\max =32.581$ \\
Convergence: & $t_{1}=5.62$ and $t_{2}=5.31$ \\
\hline
\end{tabular}

As before, offset initially occurs with trajectories and then convergence occurs quite quickly. As highlighted in Figures 8 and 9, in order to achieve close tracking in either case, quite moderately sized controls are required. Again a large amount of chattering is also evident with a greater amount observable in $u_{2}$.

The time in which convergence is obtained is comparable to that for Simulation 5.1. Initially angular velocities of 0.3 and 0.5 were tried for link 2 until using the value of 0.1 as their use resulted in large controls being required to obtain convergence. An initial angular velocity of 0.3 results in $u_{\min }=609.698$ and $u_{\max }=-2152.327$ whereas a value of 0.5 resulted in 


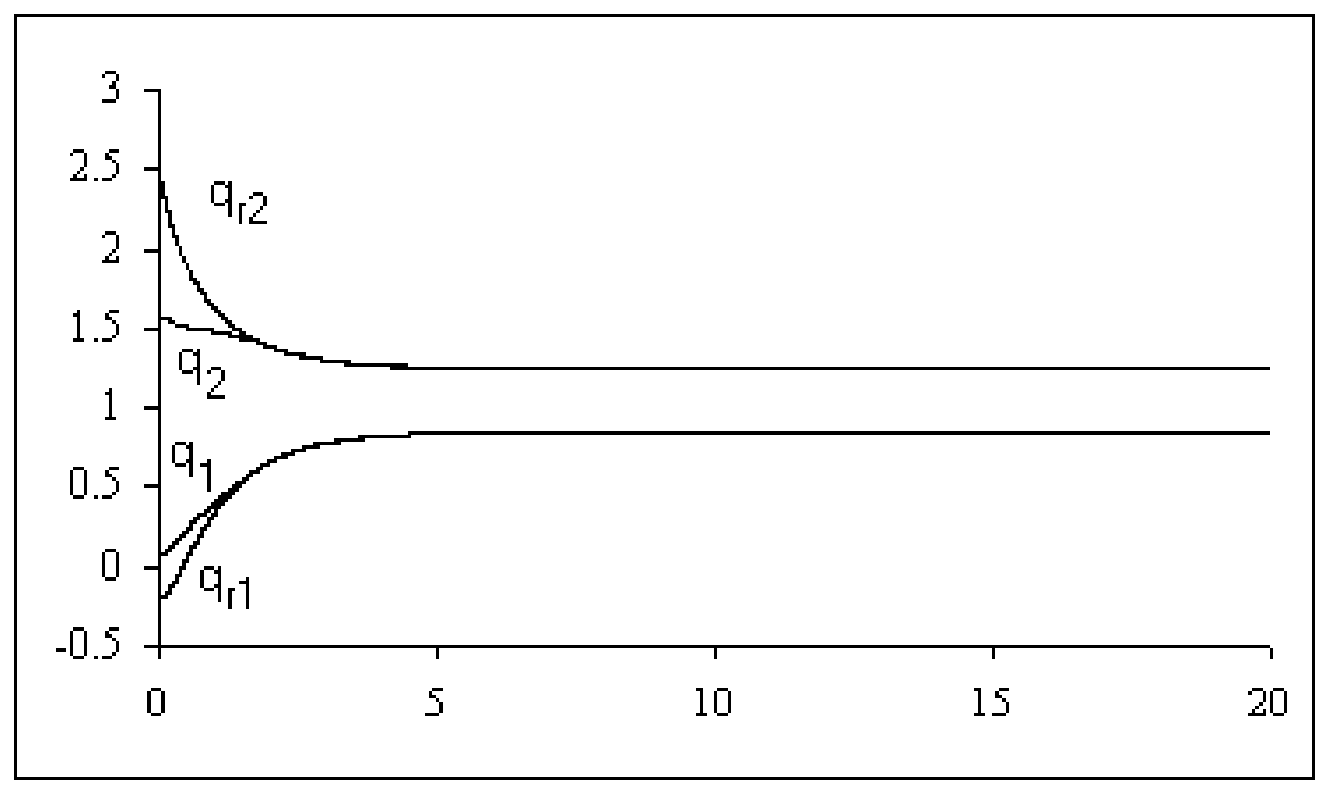

FiguRE 7: Simulation 5.2 Trajectories $q_{1}$ tracking $q_{r 1}$ and $q_{3}$ tracking $q_{r 2}$ 


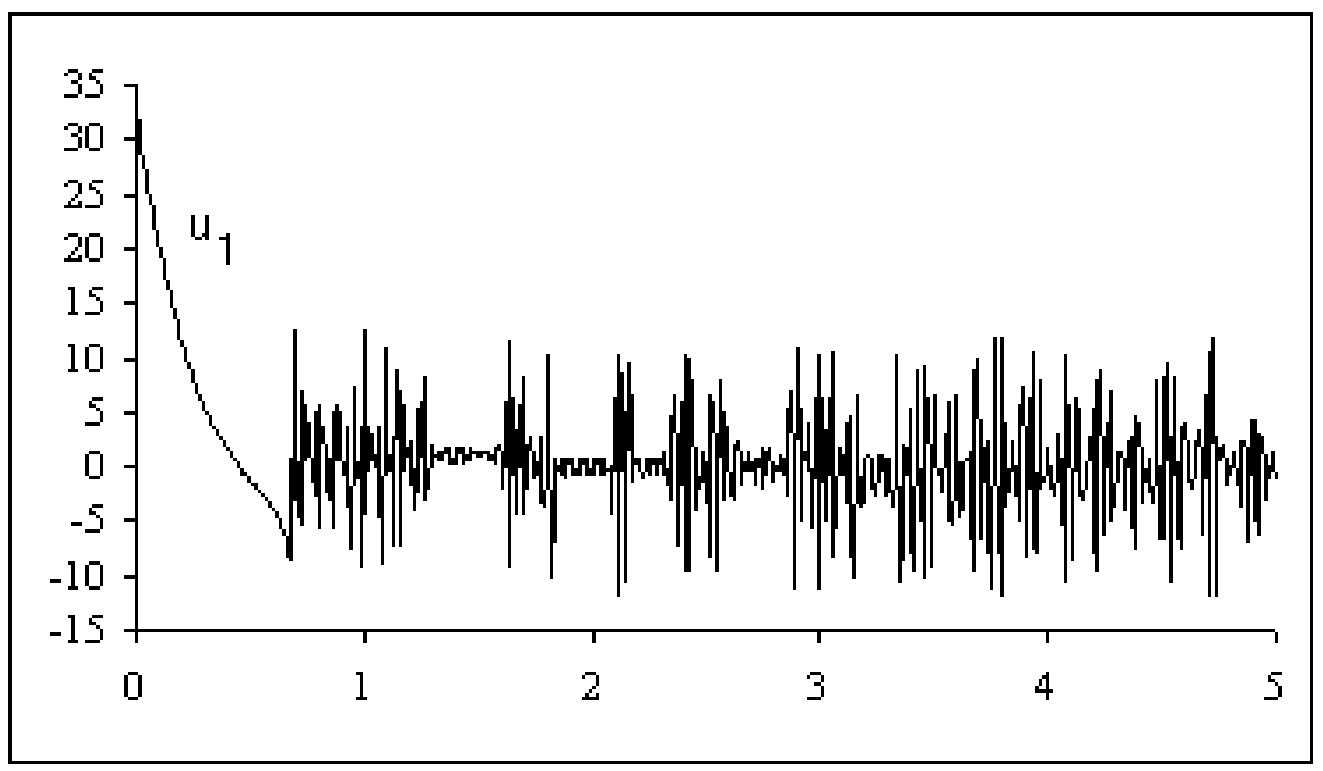

Figure 8: Simulation 5.2 control $u_{1}$ for 5 time units 


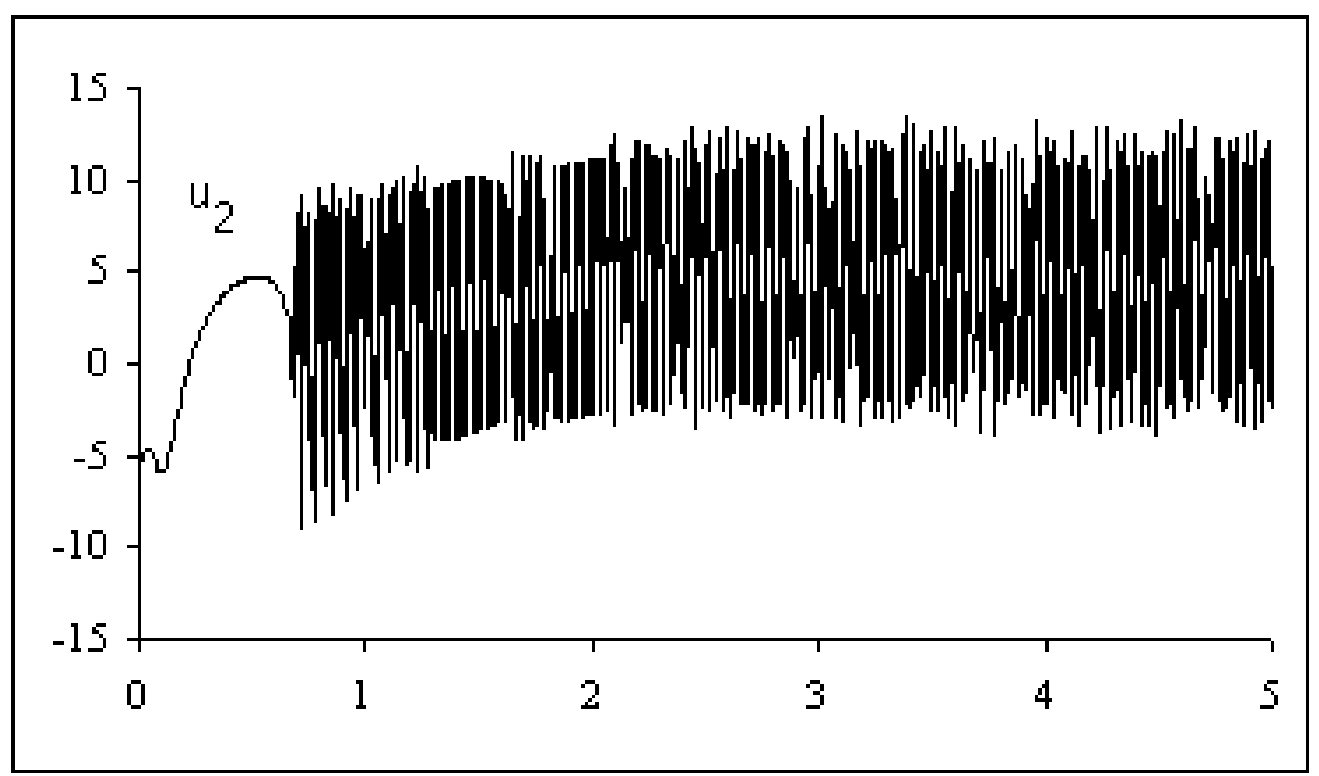

Figure 9: Simulation 5.2 control $u_{2}$ for 5 time units 


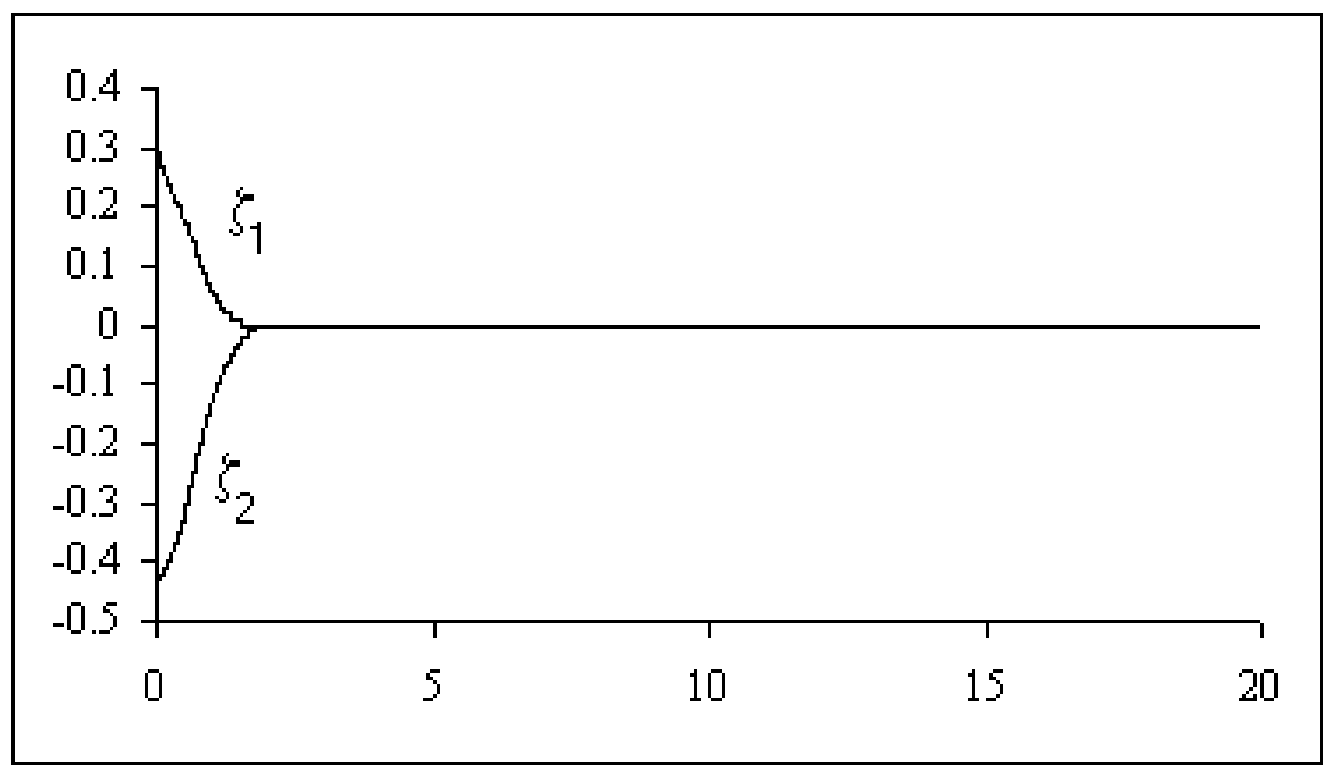

Figure 10: Simulation 5.2 Tracking errors $\epsilon_{1}$ and $\epsilon_{2}$

$u_{\min }=115.282$ and $u_{\max }=-178.977$. The range of controls required to enable the manipulator to operate within close proximity to the boundaries of $q_{1}$ and $q_{2}$ are comparable to those in Simulation 5.1. Note $\gamma_{3}$ has needed to be raised to 1000 so as to provide enough influence to enable movement away from the upper bound of $q_{2}$. 


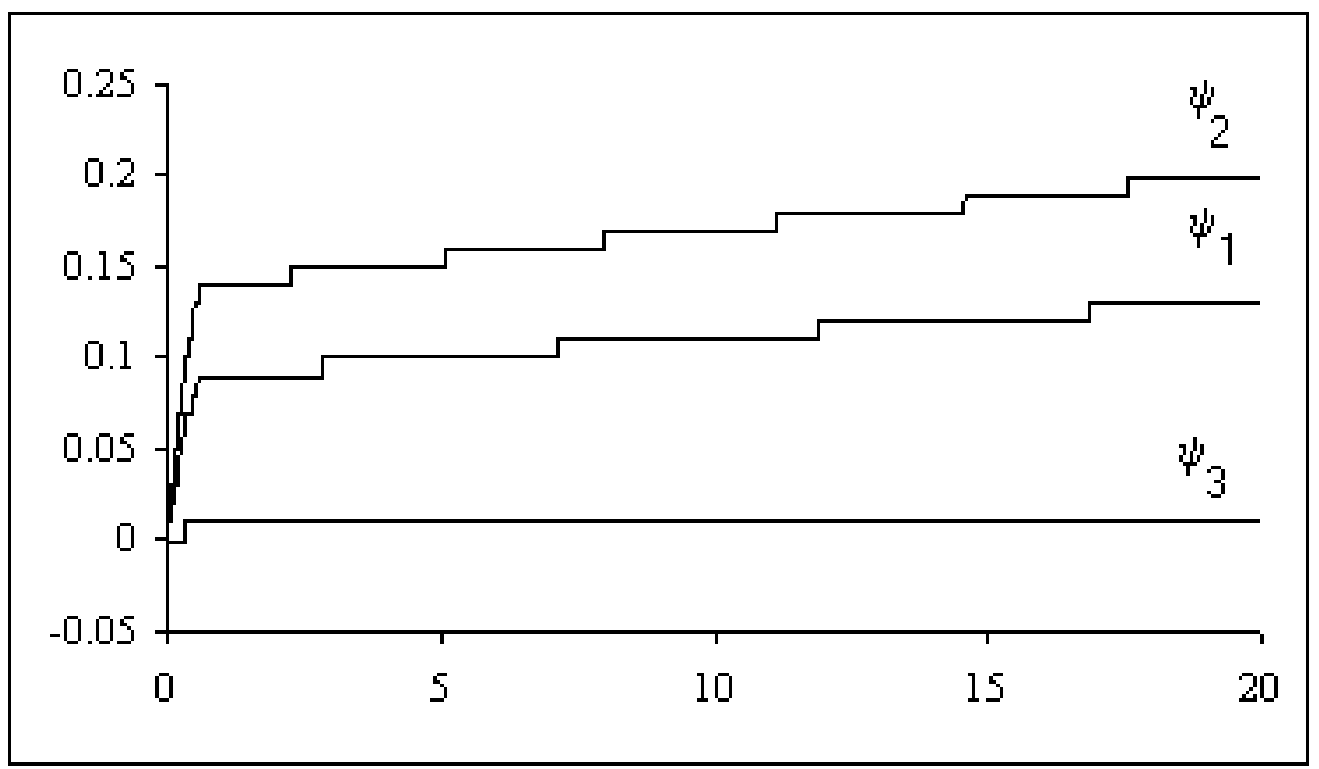

Figure 11: Simulation 5.2 Parameter error estimates $\psi_{1}, \psi_{2}, \psi_{3}$ 


\subsection{Simulation C: tracking a fixed point reference sig- nal}

This simulation again highlights the operation of the manipulator when tracking to a steady state value; $q_{1}$ and $q_{1}$ converge to 0.85 and 1.25 respectively but its initial angular position is set close to the upper boundary of $q_{1}$ and is moving toward this upper boundary with an initial angular velocity of 1.2 while its initial angular position is set close to the lower boundary of $q_{2}$ and is moving toward its lower boundary with an initial angular velocity of -0.3 . Investigations of the resulting control magnitudes and parameter assignment are conducted to ascertain how well convergence is achieved when the manipulator is operating in its boundary regions as outlined.

The initial state values of the system were selected as:

$$
\left[q_{1}(0) q_{2}(0)\right]^{T}=\left[\begin{array}{lll}
0.84 & 0.1
\end{array}\right]^{T} \text { and }\left[\dot{q}_{1}(0) \dot{q}_{2}(0)\right]^{T}=\left[\begin{array}{ll}
1.2 & -0.3
\end{array}\right]^{T} .
$$

The initial parameter values and the initial parameter estimates were set as

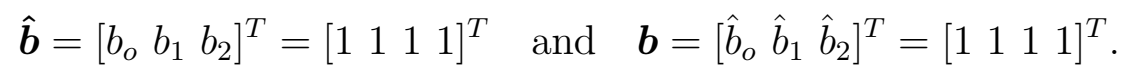

The avoidance parameters adopted were

$$
\gamma_{1}=35, \quad \gamma_{2}=10, \quad \gamma_{3}=2, \quad \gamma_{4}=1
$$

This simulation resulted in: 


\begin{tabular}{ll}
\hline Final Time: & 20 time periods \\
Final Parameter Estimates: & $\hat{\boldsymbol{b}}=\left[\begin{array}{lll}\hat{b}_{0} & \hat{b}_{1} & \hat{b}_{2}\end{array}\right]^{T}=\left[\begin{array}{lll}1.33 & 1.35 & 1.21\end{array}\right]^{T}$ \\
Controls: & $\min =-54.278$ and $\max =17.571$ \\
Convergence: & $t_{1}=5.64$ and $t_{2}=5.27$ \\
\hline
\end{tabular}

The time in which convergence is obtained is comparable to that for Simulation 5.1 and 5.2. Quite a large initial angular velocity has been chosen and it did not provide any major problem in obtaining convergence. The range of controls required to enable the manipulator to operate within close proximity to the boundaries of $q_{1}$ and $q_{2}$ are comparable to those in Simulation 5.1 where initially the manipulator is operating centrally in its workspace. Chattering is observed in both controls but is more prevalent in $u_{2}$. Interestingly $\gamma_{3}$ is required to be increased slightly to ensure convergence.

\subsection{Simulation D: tracking an oscillatory reference sig- nal}

This simulation highlights the operation of the manipulator when tracking an oscillating reference signal. The initial angular position of $q_{2}$ is set close to its upper boundary and is initially stationary while the initial angular position of $q_{1}$ is set close to its lower boundary and is away with an initial angular velocity of 0.1 . Investigations of the resulting control magnitudes and parameter assignments are conducted to ascertain how well convergence 


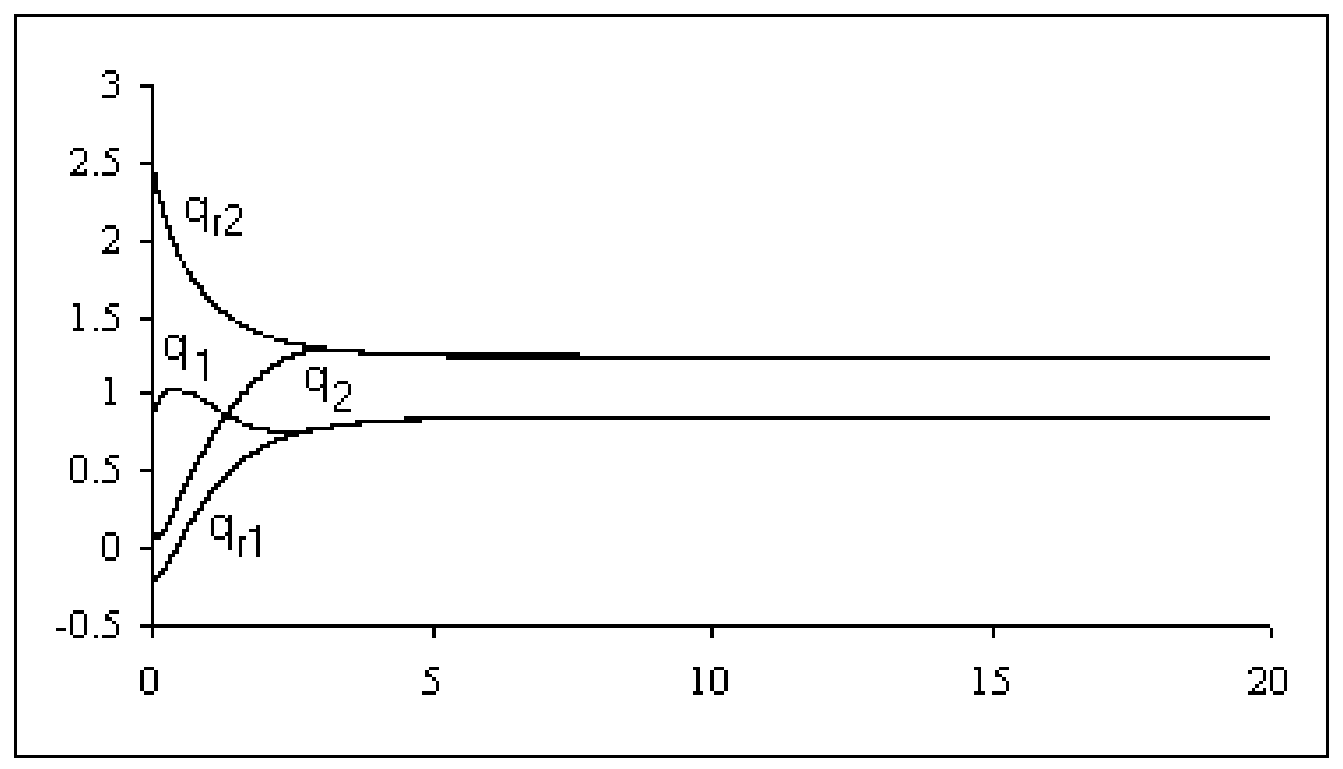

FIGURE 12: Simulation 5.3 Trajectories $q_{1}$ tracking $q_{r 1}$ and $q_{3}$ tracking $q_{r 2}$ 


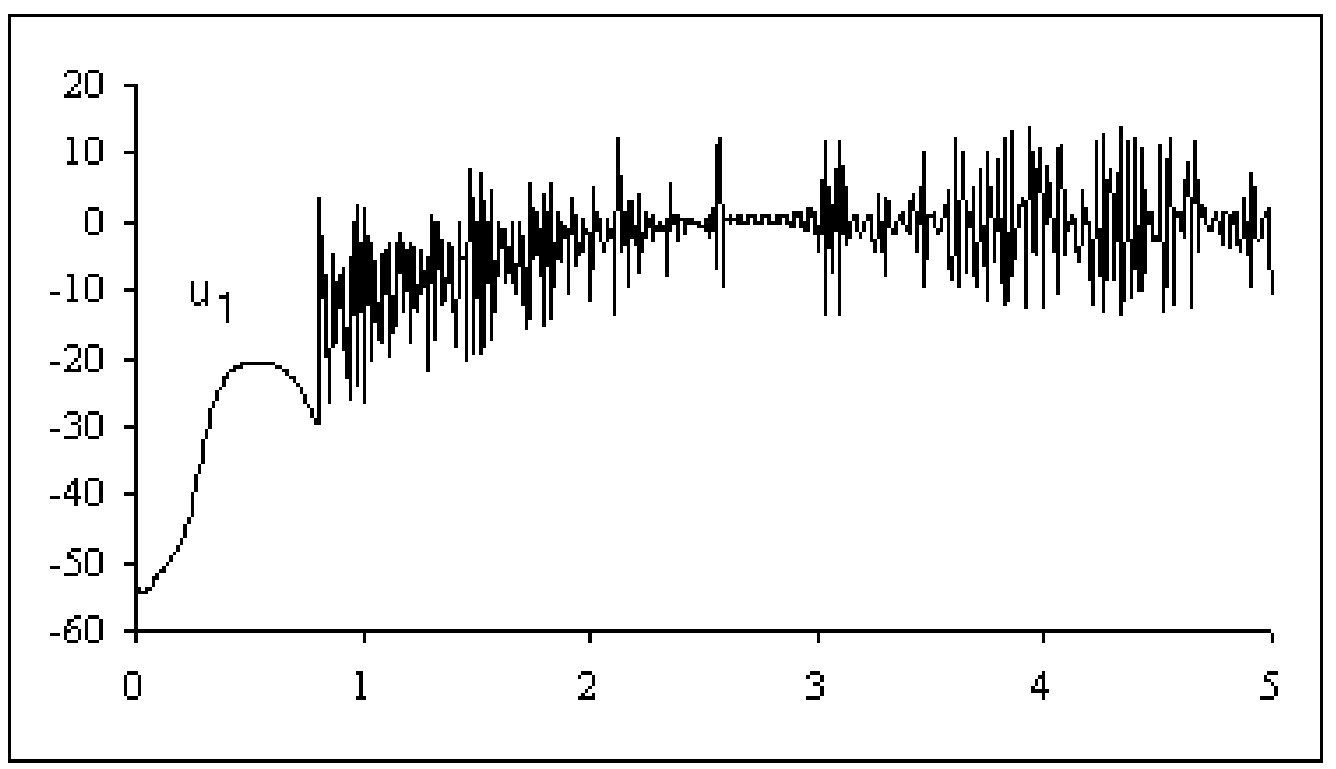

FiguRE 13: Simulation 5.3 control $u_{1}$ for 5 time units 


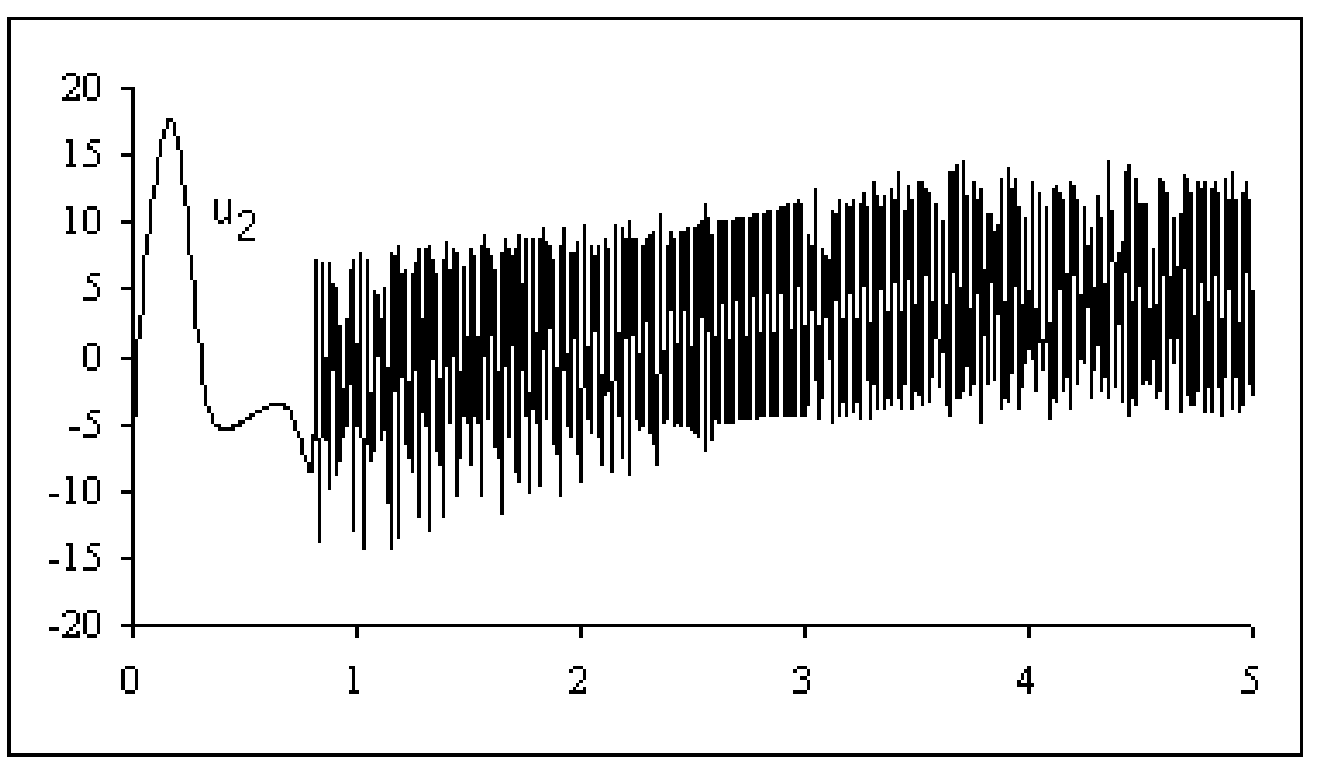

FiguRE 14: Simulation 5.3 control $u_{2}$ for 5 time units 


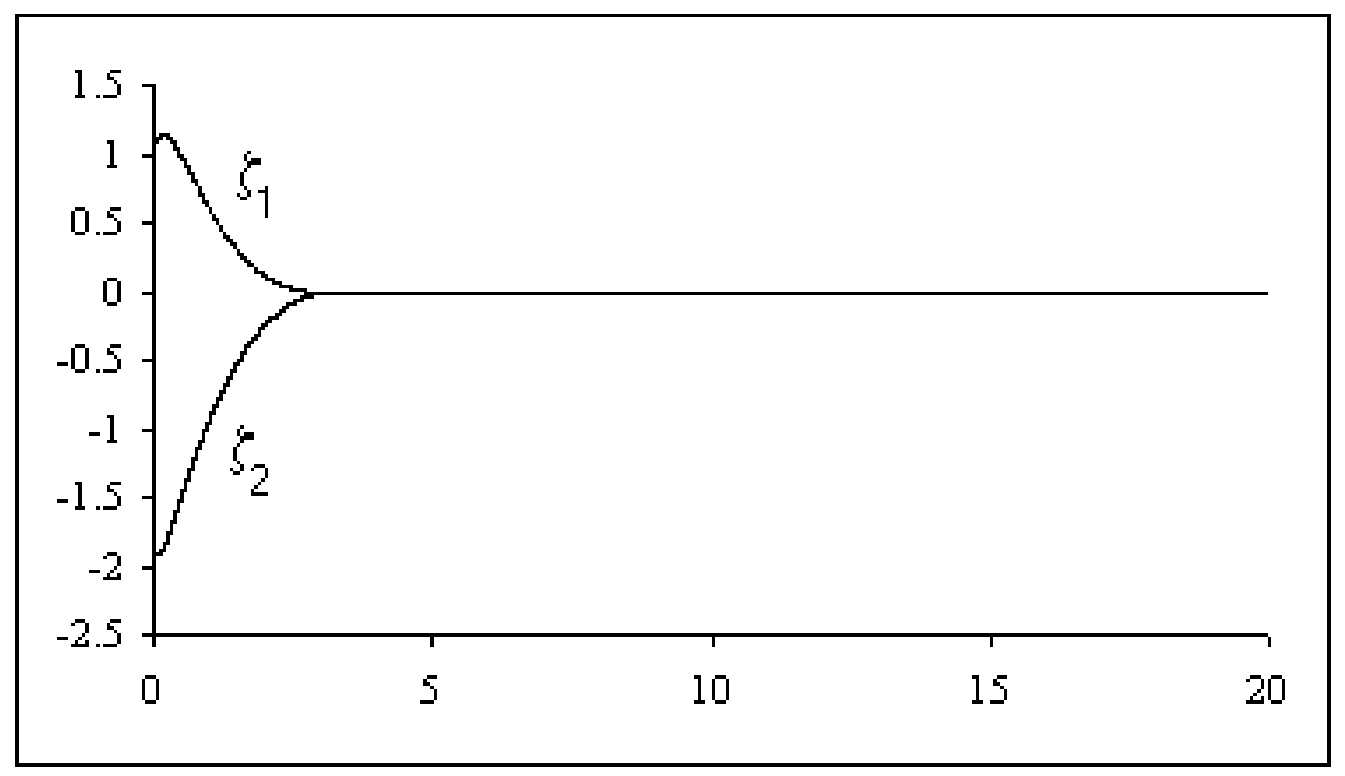

Figure 15: Simulation 5.3 Tracking errors $\epsilon_{1}$ and $\epsilon_{2}$ 


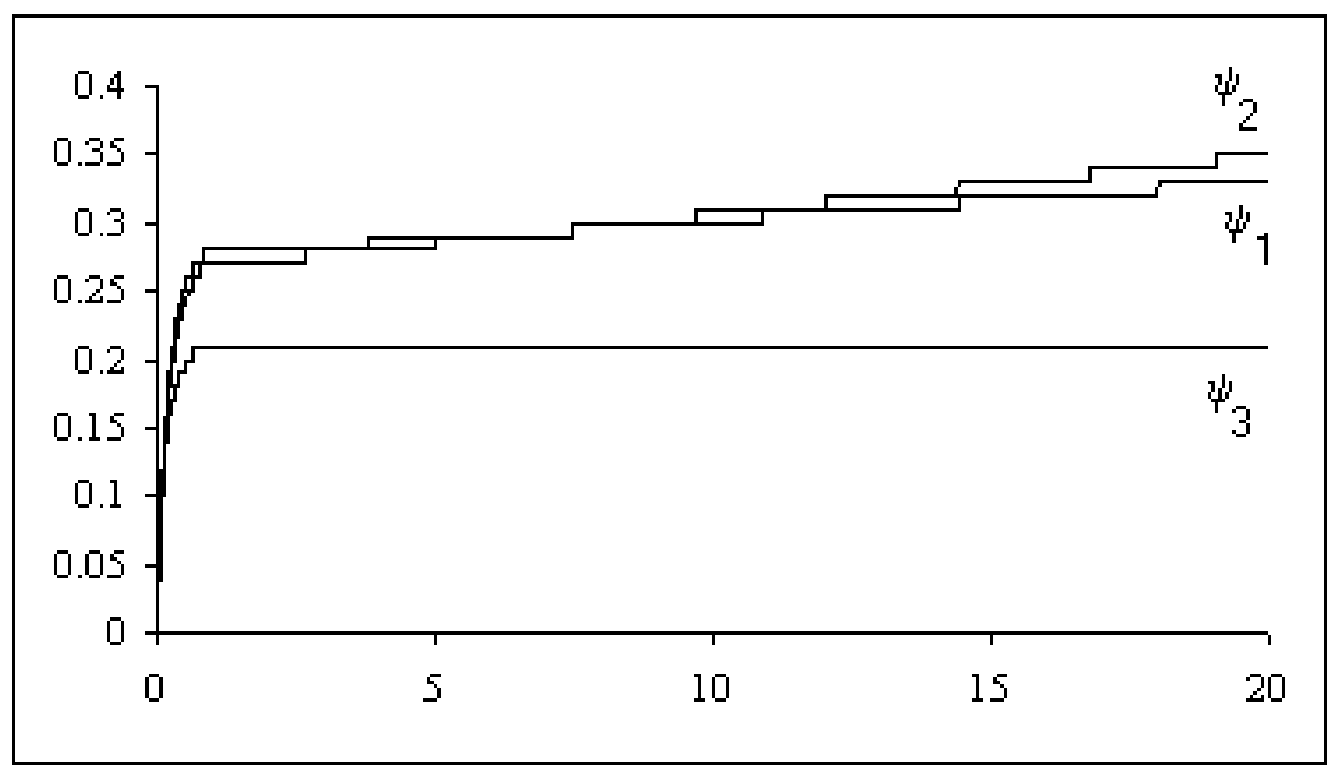

Figure 16: Simulation 5.3 Parameter error estimates $\psi_{1}, \psi_{2}, \psi_{3}$ 
is achieved and compared to that achieved for Simulations 5.1, 5.2 and 5.3 where convergence is to a steady state value.

This simulation reflects the type of work undertaken in [1] that relates to the tracking of an oscillating reference signal as outlined in Example 9.3 in Chapter 9 of [5]. In that work we adopted control based upon adaptive model reference tracking with uncertain dynamics incorporating state constraints whereas here we are implementing adaptive terminal sliding mode control with uncertain dynamics incorporating state constraints. From our investigations here we have been able to demonstrate that when using sliding mode control there has been a significant reduction in the size of the controls necessary to achieve convergence.

Here the desired trajectory reference signals were defined as

$$
q_{r 1}=0.175(1-\cos (2 \pi t))+0.175 \quad \text { and } \quad q_{r 2}=0.22(1-\cos (2 \pi t))+0.22 \text {. }
$$

This correlates to confining $q_{r 1}$ so that it has movement of freedom between 0.175 and 0.525 radians and $q_{r 2}$ has movement between 0.22 and 0.66 radians.

The initial state values of the system were selected as:

$$
\left[q_{1}(0) q_{2}(0)\right]^{T}=\left[\begin{array}{lll}
0.1 & 1.3
\end{array}\right]^{T} \text { and }\left[\dot{q}_{1}(0) \dot{q}_{2}(0)\right]^{T}=\left[\begin{array}{ll}
0.1 & 0.0
\end{array}\right]^{T}
$$

The initial parameter values and the initial parameter estimates were set as

$$
\hat{\boldsymbol{b}}=\left[\begin{array}{lll}
b_{o} & b_{1} & b_{2}
\end{array}\right]^{T}=\left[\begin{array}{llll}
1 & 1 & 1 & 1
\end{array}\right]^{T} \quad \text { and } \quad \boldsymbol{b}=\left[\begin{array}{lll}
\hat{b}_{o} & \hat{b}_{1} & \hat{b}_{2}
\end{array}\right]^{T}=\left[\begin{array}{llll}
1 & 1 & 1 & 1
\end{array}\right]^{T} .
$$


The avoidance parameters adopted were:

$$
\gamma_{1}=1, \quad \gamma_{2}=1, \quad \gamma_{3}=1, \quad \gamma_{4}=1 .
$$

This simulation resulted in:

\begin{tabular}{ll}
\hline Final Time: & 20 time periods \\
Final Parameter Estimates: & $\hat{\boldsymbol{b}}=\left[\begin{array}{lll}\hat{b}_{0} & \hat{b}_{1} & \hat{b}_{2}\end{array}\right]^{T}=\left[\begin{array}{lll}1.20 & 1.22 & 1.34\end{array}\right]^{T}$ \\
Controls: & $\min =-98.94$ and $\max =64.46$ \\
Convergence: & $t_{1}=0.0$ and $t_{2}=1.8$ \\
\hline
\end{tabular}

The choice of oscillating reference signal for $q_{r 2}$ highlighted that the range of movement of link 2 had to be greatly reduced compared to that being used for the previous simulations where tracking to a steady state was being undertaken. Even making the minor increase in the range of movement of link 2 from 0.25 to 0.75 radians resulted in the constraint $V_{4}=1.571-x_{3}$ being broken. Also an initial value of $\dot{q}_{2}=-0.4$ resulted in this constraint being broken. Once the value for $\dot{q}_{2}$ was reduced to zero convergence occurred successfully.

The tracking of $q_{1}$ to the desired trajectory of $q_{r 1}$ occurs essentially immediately while $q_{2}$ is initially offset but quite quickly tracks to $q_{r 2}$. These convergence times are much faster than that achieved in Simulations 5.1, 5.2 and 5.3.

The size of controls are significantly larger for tracking an oscillating reference signal than when tracking to a steady state value. 


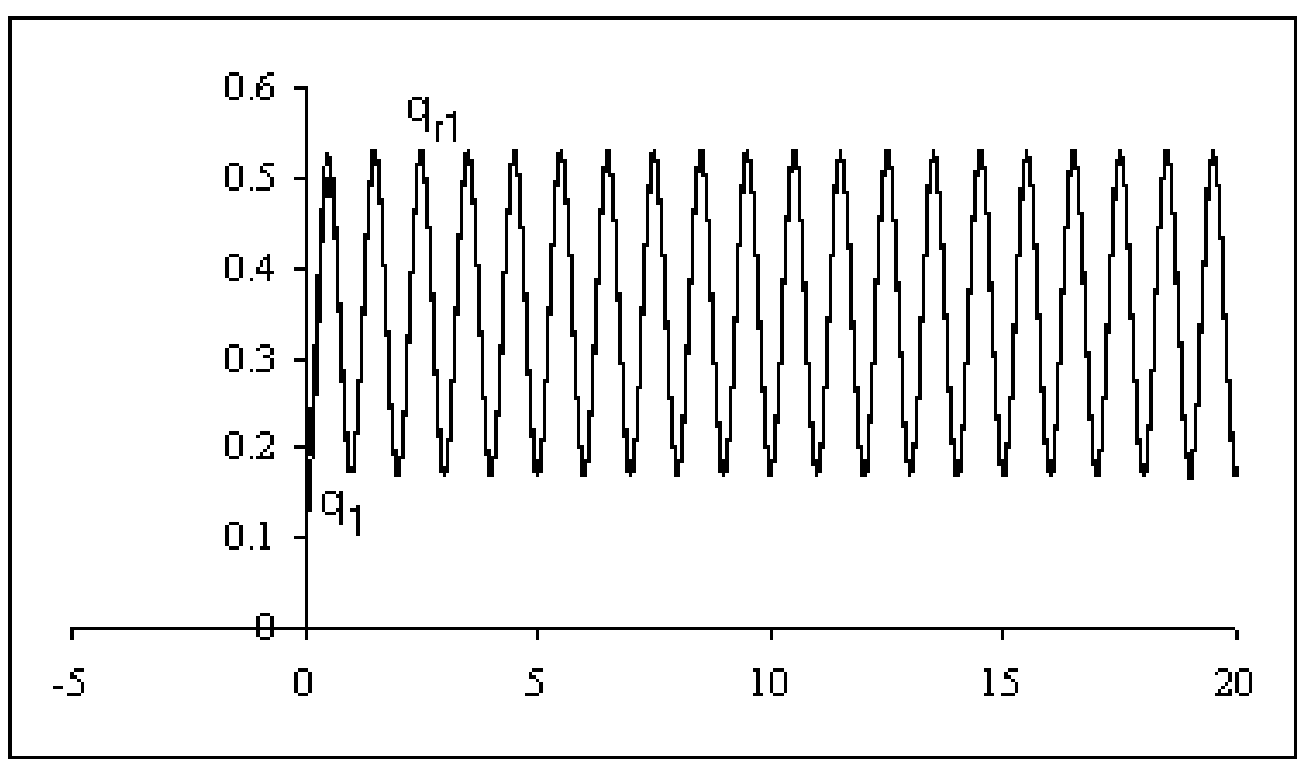

Figure 17: Simulation 5.4 Trajectories $q_{1}$ tracking $q_{r 1}$ 


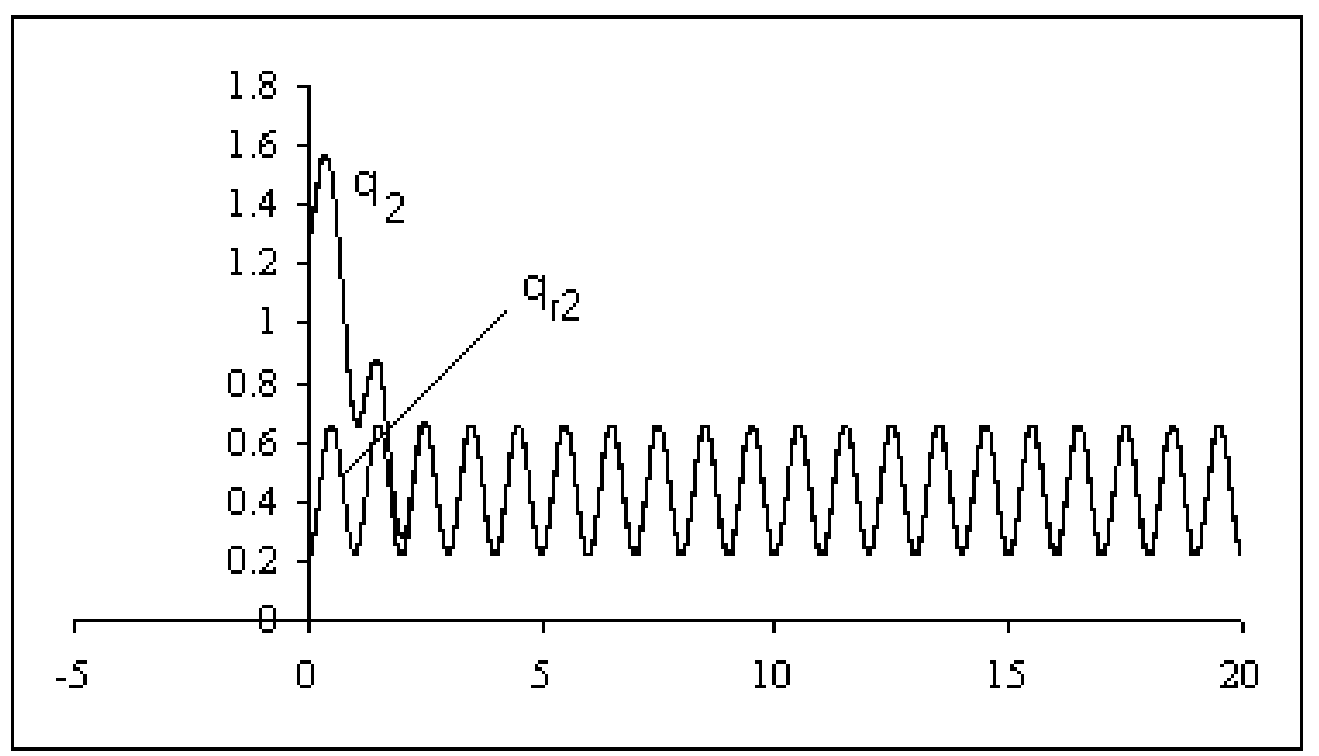

FiguRE 18: Simulation 5.4 Trajectories $q_{3}$ tracking $q_{r 2}$ 


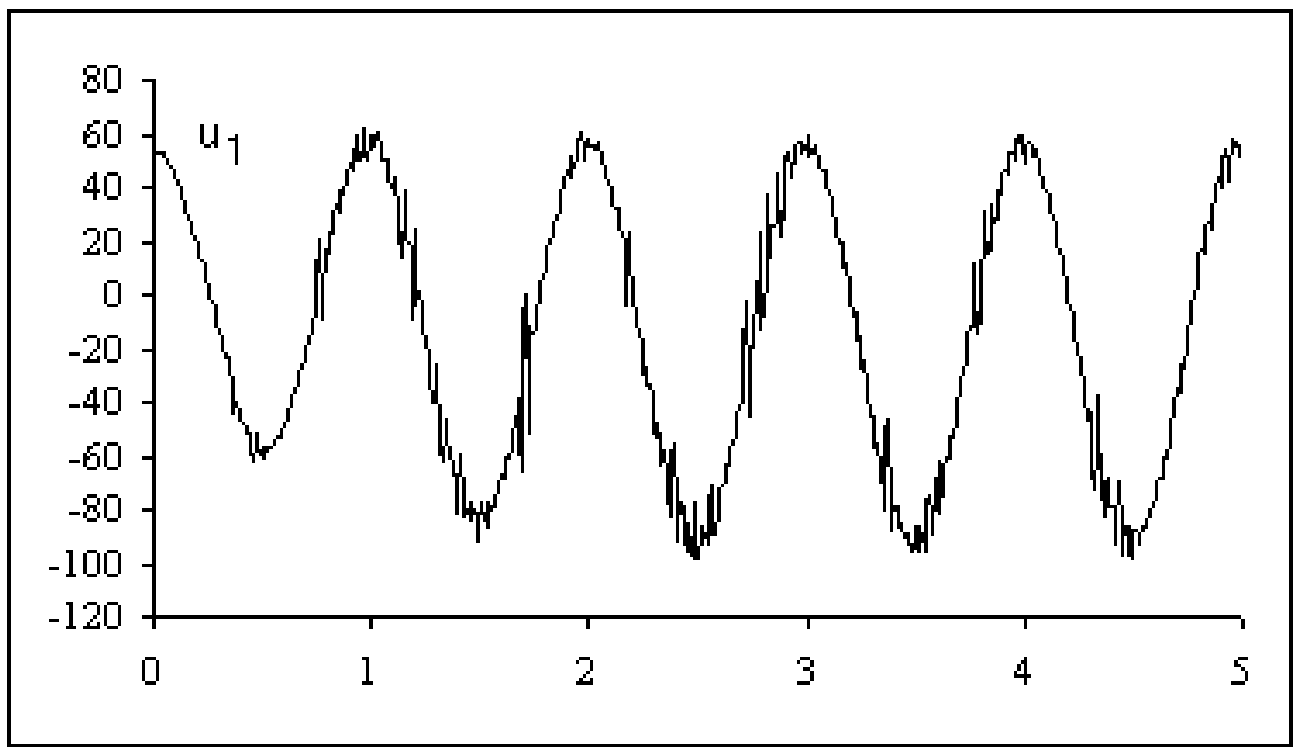

Figure 19: Simulation 5.4 control $u_{1}$ for 5 time units 


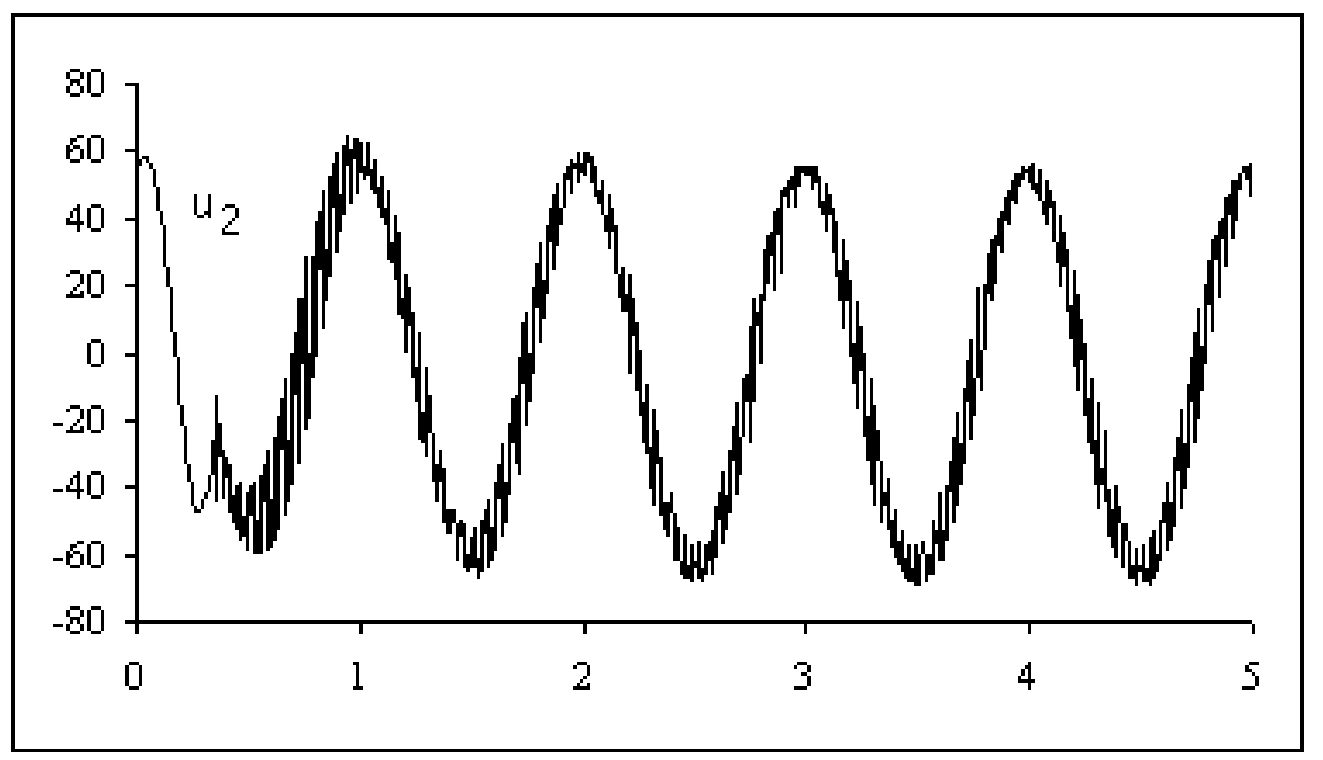

FIGURE 20: Simulation 5.4 control $u_{2}$ for 5 time units 


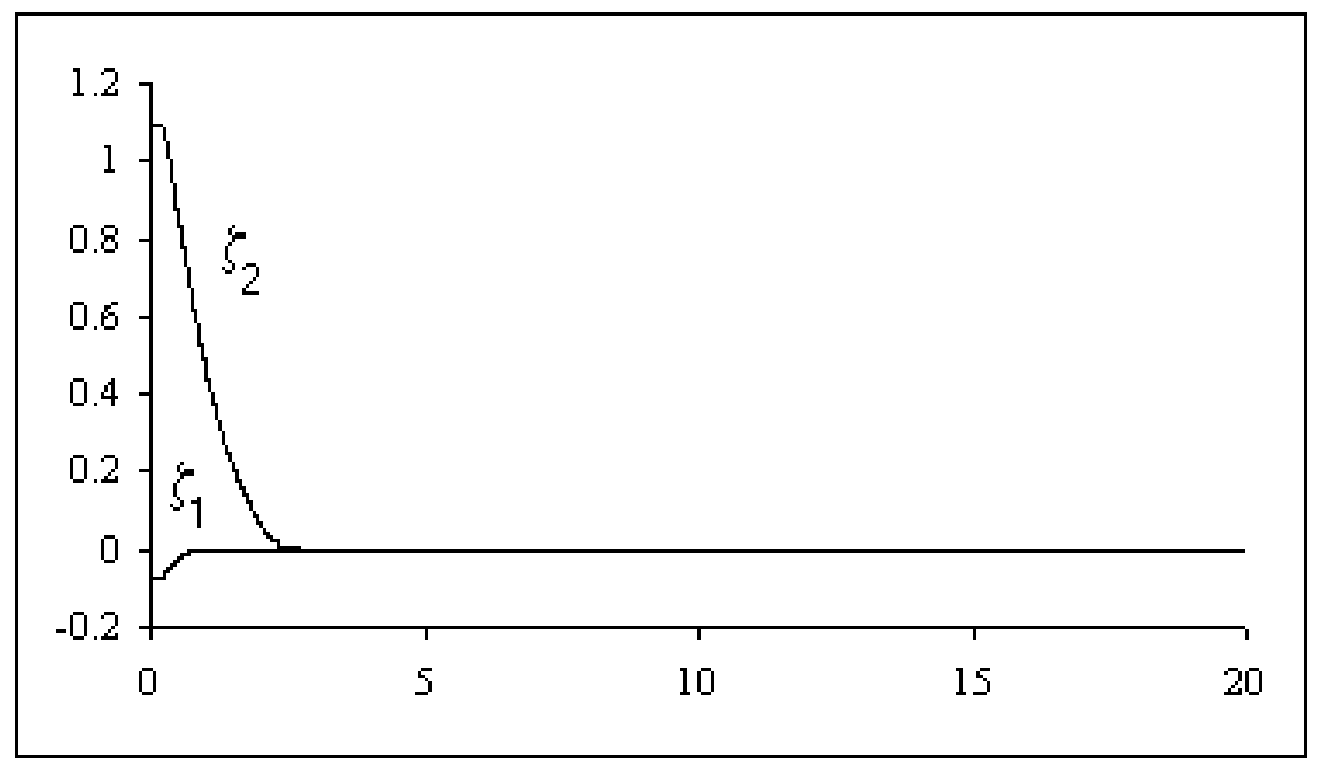

FiguRE 21: Simulation 5.4 Tracking errors $\epsilon_{1}$ and $\epsilon_{2}$ 


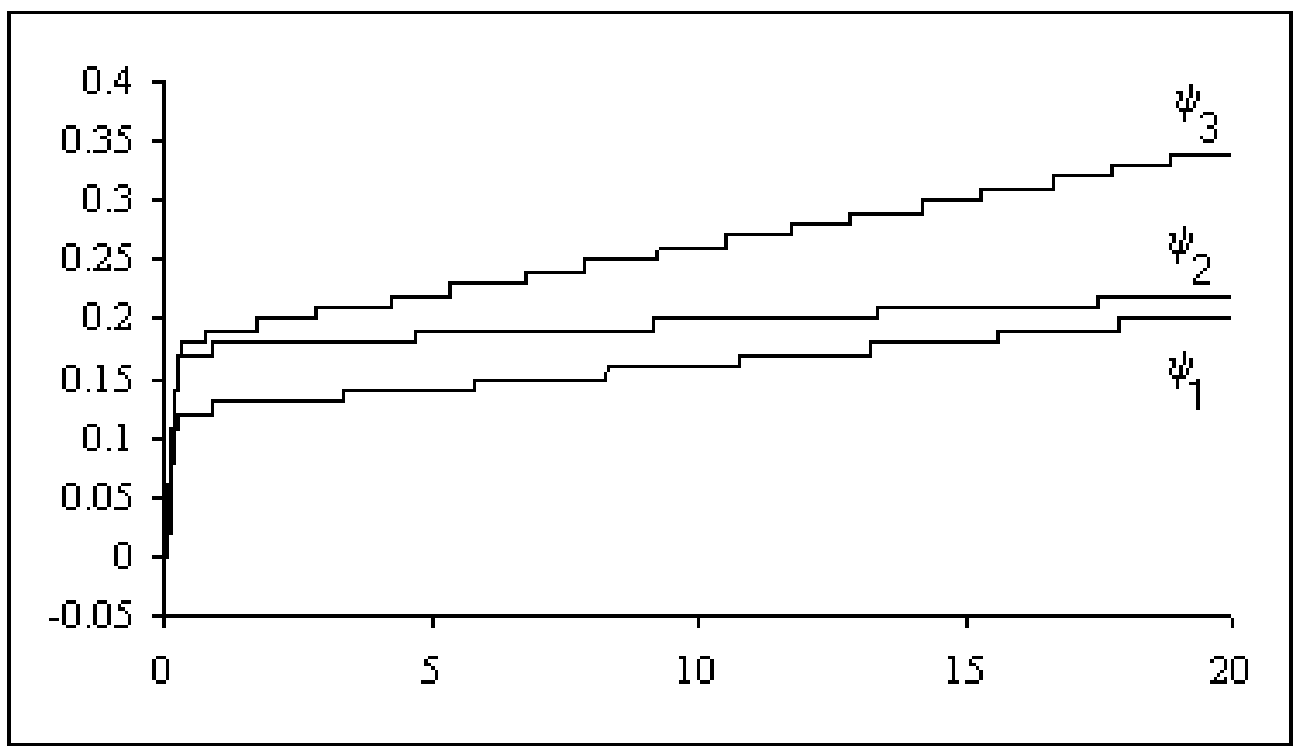

Figure 22: Simulation 5.4 Parameter error estimates $\psi_{1}, \psi_{2}, \psi_{3}$ 


\section{Conclusions}

Zhihong's [12] analysis, while achieving tracking of a desired trajectory, places no bounds upon $q_{1}$ and $q_{2}$ and in so doing does not take into consideration whether the manipulator is operating within its physical limits. We have been able to demonstrate that our technique is successful for tracking to a steady state value as well as tracking to an oscillating reference signal.

In this paper we have illustrated how to incorporate constraints using a Liapunov function to obtain similar trajectory results to [12]. This extension of Zhihong's work, with the inclusion of physical constraints upon the manipulator, enables a more realistic analysis of the physical angular motion of the manipulator in real space. Furthermore using the forms of the control laws we have developed, by incorporating state constraints, we have shown that the manipulator can successfully operate in close proximity to its boundary regions with small numerical assignments being made to gamma parameters.

\section{References}

[1] P. Keleher and R.J. Stonier "Adaptive Model Reference Tracking of a Robot Arm With Uncertain Dynamics Incorporating State Constraints", Submitted for consideration for publication in, Journal of Dynamics and Control. E143 
[2] L. Laib, "Adaptive Output Regulation of Robot Manipulators Under Actuator Constraints", IEEE Trans. on Robotics and Automation, Vol. 16, No. 1, pp29-35, 2000. E105

[3] N. Rouce, P. Habets, and M. LaLoy, "Stability Theory by Liapunov's Direct Method" Springer-Verlag, New York, 1977. E105

[4] R.J. Schilling, "Fundamentals of Robotics: Analysis and Control", Prentice-Hall, Englewood Cliffs, pp234-256, 1990. E104

[5] J. J. E. Slotine, and L. Weiping, "Applied Nonlinear Control", Prentice-Hall, Englewood Cliffs, pp40-154, 1991. E104, E105, E143

[6] R. J. Stonier, "Use of Liapunov Techniques for Collision-Avoidance of Robot Arms", Advances in Control and Dynamical Systems, Vol. 34, No. 2, pp185-215, 1990. E105

[7] R. J. Stonier and X. H. Yu, "Finite Time Switching Control for Collision-Avoidance of Mobile Robots", Proc. of the 4th International Conference on Control, Automation, Robotics and Computer Vision ICARCV'96, Singapore, pp1140-1144, December 1996. E106

[8] R. J. Stonier, P. G. Keleher, and X. H. Yu, "Finite Time Switching Control for Articulated Robots", Proc. of IEA CONTROL'97, Sydney, pp294-299, October 1997. E107 
[9] R. J. Stonier, P. G. Keleher, "Finite Time Switching Control for Two Planar PR Manipulators", Proc. of WAC98, Anchorage, ISORA 147.1-147.6, 1998. E105

[10] M. Zak, "Terminal attractors in neural networks", Neural Networks, Vol. 2, pp259-274, 1989. E105

[11] Man Zhihong, A. P. Paplinski and H. R. Wu, "A robust Mimo terminal sliding mode control scheme for rigid robotic manipulators", IEEE Trans. on Automatic Control, Vol. 39, pp2464-2469, 1994.

[12] Man Zhihong and Xinghuo Yu. "Adaptive terminal Sliding Mode Tracking Control for Rigid Robotic Manipulators with Uncertain Dynamics." Journal of S. Mechanical Engineering, Series C., Vol. 40, No. 3, 1997. E107, E108, E109, E111, E112, E115, E117, E118, E120, E121, E126, E151 